\title{
Meson bunches formed by pulsed laser-induced processes in ultra-dense hydrogen $\mathrm{H}(0)$
}

Leif Holmlid ( $\square$ holmlid@chem.gu.se )

University of Gothenburg

\section{Research Article}

Keywords: ultra-dense hydrogen, laser-induced processes, MeV particles, mesons

Posted Date: August 26th, 2021

DOI: https://doi.org/10.21203/rs.3.rs-829172/v1

License: (c) (i) This work is licensed under a Creative Commons Attribution 4.0 International License. Read Full License 


\section{Abstract}

Ultra-dense hydrogen $\mathrm{H}(0)$ (reviewed in Holmlid and Zeiner-Gundersen, Physica Scripta 2019) consists of small strongly bound molecules with interatomic distance of $0.56 \mathrm{pm}$ in spin state $\mathrm{s}=1$. It is a useful nuclear fuel for energy generation, giving heat above break-even (Holmlid, AIP Advances 2015) in laserinduced processes (Holmlid, Int. J. Hydr. Energy 2021). Nuclear processes in $\mathrm{H}(0)$ emit particles in typical meson decay chains with kinetic energy up to $100 \mathrm{MeV}$. These mesons decay and generate fast muons at up to $500 \mathrm{MeV}$ energy at current densities of several $\mathrm{mA} \mathrm{cm}^{-2}$ at 1-2 $\mathrm{m}$ distances, which corresponds to $10^{13}-10^{14}$ muons formed per laser pulse. It is shown that the mesons decay in chain processes with welldefined meson time constants in the range $10-60 \mathrm{~ns}$. The time varying signals from $\mathrm{H}(0)$ agree well with mesons $\mathrm{M}$ in decay chains as $\mathrm{A} \diamond \mathrm{M} \diamond \mathrm{N}$ where $\mathrm{N}$ is a signal muon. $\mathrm{M}$ may be a charged kaon $\mathrm{K}^{ \pm}$(decay time constant at rest $12.4 \mathrm{~ns}$ ) or a charged pion $\pi^{ \pm}$(decay time constant at rest $26 \mathrm{~ns}$ ) or a long-lived neutral kaon $\mathrm{K}_{L}^{0}$ (decay time constant at rest $51 \mathrm{~ns}$ ). Ultra-dense protium $\mathrm{p}(0)$ gives the same time constants as $\mathrm{D}(0)$ but slightly different decay-chains. The meson bunches observed are similar to the meson bunches from nucleon + antinucleon annihilation. The energy gain in the nuclear process is at least 8000 , strongly indicating baryon annihilation for which process further evidence is given in other recent publications.

\section{Introduction}

Ultra-dense hydrogen $\mathrm{H}(0)$ [1] is known to exist in several forms, as ultra-dense deuterium $\mathrm{D}(0)$ [2], ultradense protium $\mathrm{p}(0)[1,2,3]$ and as mixed phases $\mathrm{pD}(0)[4,5]$. With an interatomic distance of $2.3 \pm 0.1 \mathrm{pm}$ [6] in spin level $s=2[1,2]$, they are the densest forms of matter that have ever been studied, with a density of a few hundred $\mathrm{kg} \mathrm{cm}^{-3}$. These materials are superfluid [7] and superconductive [8] even at room temperature. Transition points to a normal non-super state exist at a few hundred $\mathrm{K}$ above room temperature [9]. These ultra-dense materials have been studied by three main methods [1], of which rotational emission spectroscopy is the most well-known method, giving accurate bond distances for spin quantum numbers $s=2,3$ and 4 with precision in the femtometer range. How can such an ultra-dense material give rise to high-energy nuclear particles? The obvious answer is via nuclear processes due to the short distance between the nuclei. The production process for this novel nuclear fuel was recently reviewed [10].

Particles in the $\mathrm{MeV}$ range are easily generated by nuclear processes using < $0.4 \mathrm{~J}$ laser pulses [11, 12].

${ }^{4} \mathrm{He}$ and ${ }^{3} \mathrm{He}$ ions have been observed by time-of-flight [13] which is a clear signature of $D+D$ fusion. Heat generation from nuclear processes above break-even was reported already in 2015 [14]. The MeV particle signal in the vacuum chamber has high intemsity. It can be measured directly by a fast oscilloscope connected to a metal collector. This gives accurate below-ns-range timing. These particles have been observed at beam distances up to $2 \mathrm{~m}[11,12]$. The particle velocity is often $>>100 \mathrm{MeV} \mathrm{u}^{-1} \mathrm{It}$ is shown $[11,12]$ that the fast particles have mass and that they penetrate through $\mathrm{mm}$ thick metal with attenuation and often a slight delay, which agrees with muons formed by meson decay. Magnetic 
deflection studies confirm that many of the initially formed particles are neutral [15], thus probably neutral kaons. The particles with MeV energy were shown to be part of a decay chain with the characteristic meson decay time constants [15-17]. Here, we show that the laser-induced particles decay with well-defined meson decay life-times to very fast charged particles i.e. mainly muons. Muons can be detected by ordinary energy spectroscopy methods from the laser-induced nuclear processes [18-20]. Accurate decay-time measurements of $2.20 \mu \mathrm{s}$ [20] confirm that these particles are muons. It is thus concluded that the decaying mesons [21] give muons as expected.

Related results on laser-induced nuclear processes exist. They mainly aim at ignition of nuclear fusion, for example by laser compression of a hydrogen fuel pellet to high pressure and temperature, as originally proposed already in the 1980's [22]. This method is used with the "world's most powerful laser" at the National Ignition Facility (NIF) in the US [23]. The amount of fusion was measured by the neutrons released, and other nuclear processes than $D+T$ fusion were not looked for. The progress in such inertialconfinement fusion (ICF) studies is still plagued by instabilities in the compressed hydrogen fusion fuel [24]. Such instabilities may be due to condensation to $H(0)$, as pointed out in Ref. [25]. Schemes using lower compression and a fast igniting laser pulse are also under development [26]. On a smaller scale, promising reports on laser-induced fusion reactions in hydrogen clusters exist [27, 28]. In laser-induced fusion reactions with a 10 TW laser [29] the neutron energy was found to be up to $4 \mathrm{MeV}$. This was suggested to be due to deuteron acceleration in the laser-produced plasma.

Some methods have been used previously to form particles with energy in the $\mathrm{MeV}$ range from hydrogen, using intense lasers. Multi-MeV particles, mainly protons, were formed in an experiment with a $2 \mathrm{~J}, 30 \mathrm{fs}$ laser at various targets [30]. The high particle energy was suggested to be due to the plasma formed. By using a $1 \mathrm{TW} \mathrm{CO}_{2}$ laser, it was in another case possible to obtain a 1-2 MeV monoenergetic proton beam from a hydrogen gas jet [31]. There exists also one report on the formation of alpha particles at MeV energy during hydrogen desorption from a complex solid phase containing the hydrogen active catalyst $\mathrm{Pd}$ [32]. Mesons have been produced in large facilities by laser-derived scattered photons at GeV energy, like $\mathrm{p}+\mathrm{y}$ giving $\mathrm{K}^{+}$and $\pi^{+}$among others [33]. Mesons can also be formed by fast protons from fusion reactions like $p+d$, for example giving ${ }^{3} \mathrm{He}$ and $\mathrm{K}^{+}+\mathrm{K}^{-}[34]$. In another case, the possibility to diagnose processes involving $B$ mesons by fast pulsed lasers was investigated [35].

\section{Theoretical Background}

Ultra-dense hydrogen $\mathrm{H}(0)$ is a quantum material. Since a review of $\mathrm{H}(0)$ was published recently [1], the description here is kept very brief. The production of $\mathrm{H}(0)$ was also recently reviewed [10]. $\mathrm{H}(0)$ is composed of molecules of various forms, mainly in a superfluid chain form $\mathrm{H}_{2 N}$ with the $\mathrm{H}-\mathrm{H}$ pairs rotating around the central axis (vortex) of the molecule [1]. Also other forms are commonly observed, like the non-superfluid small clusters $\mathrm{H}_{4}(0)$ and $\mathrm{H}_{3}(0)$ [36]. The ultra-dense states are characterized by their (mixed) spin quantum number $s=1,2, \ldots .$. [1]. The state $s=2$, which is the one formed most easily in the laboratory, has an interatomic distance of $2.3 \mathrm{pm}$. The state $s=1$ is formed from $s=2$ both 
spontaneously at a low rate [37] and by laser pulse induction [18]. In this level $s=1$ at internuclear distance of $0.56 \mathrm{pm}$ [1], it is concluded that nuclear processes are very fast. At similar D-D distances in muon-catalyzed fusion, the rate constant for fusion is $10^{9} \mathrm{~s}^{-1}$ [38]. This means that the laser energy is only required for transferring $\mathrm{H}(0)$ from $s=2$ to $s=1$. Thus, no high temperature is required for fusion or other nuclear processes, and plasma formation is indeed counterproductive for fusion but difficult to avoid. Besides, plasma formation in the experiments due to the laser pulse-impact will influence the energies of the ejected particles by collisions, giving slower particles with non-specific energies, which may decrease the energy resolution which is important for the experiments [39].

The decay-time experiment presented here require some analysis, which is repeated from previous publications [15-17]. The signal is often measured by foil collectors at two different distances in a beam ejected from the pulsed-laser interaction with ultra-dense hydrogen $\mathrm{H}(0)$. The two collector signals are quite similar in their general time behavior with a peaked signal decaying with one or a few time constants. This means that the signal is due to one or a few types of intermediate particles $\mathrm{M}$ which are formed and decay like $\mathrm{A}$ à $\mathrm{M}$ à $\mathrm{N}$. The same time dependence can be observed at both collector distances. The transverse energy given by the decay processes to the products means that some particles do not reach the outer collector. This complicates the comparison of the signals at the two collectors. See further below.

The time dependence of the intermediate $M$ is easily derived from the rate equations for $[15,16]$

The time dependence of the intermediate $\mathrm{M}$ is easily derived from the rate equations for $\mathrm{A} \stackrel{k_{1}}{\longrightarrow} \mathrm{M} \stackrel{k_{2}}{\longrightarrow} \mathrm{N}[15,16]$

$$
\begin{aligned}
-\frac{d n_{\mathrm{A}}}{d t} & =k_{1} n_{\mathrm{A}} \\
\frac{d n_{\mathrm{M}}}{d t} & =k_{1} n_{\mathrm{A}}-k_{2} n_{\mathrm{M}}
\end{aligned}
$$

as

$$
n_{\mathrm{M}}=\frac{k_{1}}{k_{2}-k_{1}} n_{\mathrm{A} 0}\left(e^{-k_{1} t}-e^{-k_{2} t}\right)
$$

where $n_{\mathrm{A} 0}$ is the number density of the precursor at time $t=0$ thus during the laser pulse. This derivation assumes that the initial number density $n_{\mathrm{MO}}$ is zero. The curve shape in Eq. (3) is used to model the results found here, and is often shown as dashed curves in the figures below. The results are given as time constants $\tau=1 / k$.

For some tests, one further step in the chain process has been used as $\mathrm{A} \stackrel{k_{1}}{\longrightarrow} \mathrm{M} \stackrel{k_{2}}{\longrightarrow} \mathrm{N} \stackrel{k_{3}}{\longrightarrow} \mathrm{O}$.

The corresponding time dependence is easily derived. It is known that the decay chains from kaons are not strictly linear but also contain other steps $[21,40,41]$. When the signal agrees with Eq. (3), a linear 
chain is approximately valid. Departures from this form may be due to competing steps or due to two different chains or steps giving the same intermediate. The influence of other time effects like the laser pulse width will be discussed below.

$\mathrm{H}(0)$ clusters can take part in nuclear processes which give particles with energies corresponding to $>100$ $\mathrm{MeV} \mathrm{u}^{-1}[15-17]$. This means that the processes observed are not ordinary fusion processes which can only give a few MeV energy. A study of the particles ejected may give evidence of the type of processes involved, which is the motivation for the present study. The basic nuclear processes are thus of no concern for the present study, only the meson formation and decay processes. A possible model is that two baryons for example one baryon and one antibaryon (formed from a bayon by beta capture) annihilate to form three pairs of light mesons (kaons and pions) [21,40]. Detailed descriptions of the annihilation process were recently published $[39,42]$. The energetics is exact at a level of $0,4 \%$ using measured kinetic energies for the mesons. It is observed in the present and earlier studies that the particles ejected decay, with the characteristic decay-times of kaons and pions. This was published previously $[15-17,39,42]$. For example, the laser-induced processes giving the decay are schematically

$$
\mathrm{H}_{N}(0) \rightarrow . . \rightarrow \ldots \rightarrow \mathrm{K}^{ \pm} \rightarrow \pi^{ \pm} \rightarrow \mu^{ \pm} \rightarrow \mathrm{e}^{ \pm}
$$

also giving photons and other leptons like neutrinos $[40,41]$ which are not shown in this reaction sequence. The signal observed at the collectors is mainly due to muons from the decay of kaons and pions. These muons eject electrons from the collectors. The long lifetime of the muon product $\mu^{ \pm}$of $2.20 \mu \mathrm{s}$ means that muons do not decay inside the apparatus but escape through the walls [20] or may get captured in the walls if the muons are negative. The muons formed in the experiments have been measured in several studies by ordinary energy spectroscopy methods $[18,19,37]$. A full description of the muon decay including the differences between positive and negative muons in these experiments is published [20].

\section{Experimental}

The layout of the apparatus is shown in Fig. 1. A pulsed Nd:YAG laser is focused onto a metallic target plate with a lens of $40 \mathrm{~cm}$ focal length. The laser is used with $532 \mathrm{~nm}$ light and $5 \mathrm{~ns}$ pulse length at maximum $120 \mathrm{~mJ}$ pulse energy. This gives a nominal spot size of $30 \mu \mathrm{m}$ for a Gaussian beam and a power density of $<3 \times 10^{12} \mathrm{~W} \mathrm{~cm}^{-2}$. The stainless steel laser target in the center of the main vacuum chamber is covered by a thin super-fluid layer of $\mathrm{D}(0)$ or $\mathrm{p}(0)$ [7]. The source for ultra-dense hydrogen $\mathrm{H}(0)$ was described previously [6]. In it, a potassium-doped iron oxide catalyst sample [43, 44] forms $\mathrm{H}(0)$ from deuterium gas $(99.8 \%)$ or natural hydrogen $(99.9995 \%$ pure hydrogen, naturally containing only $0.016 \%$ D) at a pressure close to $1 \times 10^{-5}$ mbar. The catalyst is a porous iron oxide cylinder that fills the opening of the gas feed tube 1-2 cm above the laser target. Thus the laser beam does not impact on the catalyst and no ablation from the catalyst exists. The impact angle of the laser beam on the target is $45^{\circ}$ towards the normal, and the signal is taken out to the collectors at a direction of approximately $60^{\circ}$ relative to the 
normal. The signal is thus observed in a quite arbitrary direction relative to both the laser beam and the target normal.

Two metal collectors in a separately pumped chamber are used here as seen in Fig. 1. The inner collector is at $64 \mathrm{~cm}$ distance from the target and can be rotated out from the beam to the outer collector. This inner collector consists of a stainless steel circular plate with an opening in the middle. This structure and opening is covered with three Al foils each with thickness $20 \mu \mathrm{m}$ thus a total Al thickness of $60 \mu \mathrm{m}$. This collector is mounted on an arm behind an annular plate covering the entire diameter of the tubular chamber and with a central opening of $38 \mathrm{~mm}$ diameter. The outer collector is of a similar construction and is at either 103 or $163 \mathrm{~cm}$ distance from the target. Thin foils are preferred as collectors since they do not give so much interaction with the muons in the beam, thus giving less pair formation [12] and muon capture and decay processes [45]. This simplifies the interpretation of the signals. The signal to the collectors is measured by a fast digital 2-channel oscilloscope (Tektronix TDS $3032,300 \mathrm{MHz}$, rise-time $1.3 \mathrm{~ns}$ ) with $50 \Omega$ input resistance, not using any preamplifier. The rise-time of the signal in the figures below is as short as $3 \mathrm{~ns}$ and thus equal to the laser pulse rise time. This proves that no limiting time constant is due to the measuring circuit. Thus, the characteristic meson-decay time constants observed close to 13,26 , and 52 ns are not due to any measurement error. No noise from the laser influences the results, as can be directly verified in the experiments by closing the valve to the detector part at the wall of the laser target chamber. The normal peak noise from the very quiet laser to the collectors is below $1 \mathrm{mV}$. Digital averaging (512 repetitions) is used for all the spectra to remove unrelated high-frequency noise. The shot to shot variations of the signals are small. The bias voltage used on the collectors is either given by a shielded $24 \mathrm{~V}$ or $50 \mathrm{~V}$ battery or by a DC power supply for higher voltages. The pulse signal is in the high voltage case taken out through a $1 \mathrm{nF} \mathrm{HV}$ capacitor. The oscilloscope trigger is from a photo-diode close to the laser. The cabling lengths from the trigger and the collectors are adjusted so that the trigger point on the oscilloscope is close to the true zero at the laser pulse impact on the target. Many timing results involve only the difference in time between the two collectors. Since the signals are measured by moving the same cable between the collectors, no zero time error influences such results.

\section{Results}

Most of the results here concern the processes that form the mesons and muons, while the identification of the different mesons from their decay-times have been published previously (as cited in the Appendix) and further high-precision results have been published [39]. In the figures, calculated curves are shown which are intended to be helpful for the understanding of the meson processes, but they are not fitted to the signals and not intended for extracting high-precision life-times. Such results are already published [39]. This approach is necessary since so many different mesons are formed (7 different meson types in all). They are decaying simultaneously, giving overlapping muon signals at the collectors. Of course, the full processes are better observed in the present experiments. Since the understanding of the processes is the aim as successfully demonstrated in [39], direct experiments are always better than modeling calculations which may find secondary processes at best. High-precision results on the decays have been published [39] and are now summarized in the Appendix. 


\subsection{General particle properties}

Here, we first verify once more that the particles observed have a mass. In Fig. 2, an experiment with two collectors in line is shown. By recalculating the time base to compensate for the different collector distances, a fairly good agreement between the signals to the two collectors is found, which shows that the signal in this case is due to particles with mass and with a distribution of velocities. The particles do not change their velocities during transit very much in this case. Thus, the signal is clearly not due to any kind of photons which would have a common velocity. Neutrons are excluded since they cannot be detected by the thin foil collectors. The slightly imperfect matching of the two curves in Fig. 2 indicates only a small change in the flux properties during transit.

These particles penetrate to some extent through the $60 \mu \mathrm{m}$ thick Al foil in the inner collector. This is shown in Fig. 3, where the signal at the outer collector at distance $163 \mathrm{~cm}$ is shown when the inner collector is both open (red) and closed (black). Between 5 and $10 \%$ of the particle flux penetrates (the ratio of the left-hand and right-hand vertical scales) without considerable deflection, and continues for 1 $\mathrm{m}$ along the beam to the outer collector. With positive bias on the collector, secondary electrons from the walls in the apparatus may be collected. This may distort the signal. With zero and negative collector bias, a short decay is observed. There is also a clear delay of the peak of the intensity in Fig. 3, and also a loss of slower flux. Thus, the particles have mass and are slowed down or deflected by collisions in the foil. The particles are not photons which could not be delayed as observed.

The signals observed by the collectors are from a very small angular range around a main beam direction of approximately $60^{\circ}$ from the target normal, and at $90^{\circ}$ from the laser beam. This direction is arbitrary and the inner collector covers a small angular range around it, approximately $1 \times 10^{-3} \mathrm{sr}$, while the outer collector covers only $1.5 \times 10^{-4} \mathrm{sr}$. This means that the intensity is expected to be quite isotropic in the range that the collectors cover. As seen in Fig. 4, the signals at the inner and outer collectors have different time distributions such that the signal at the outer collector appears much faster than a particle observed at the inner collector. If the signal was due to particles moving at relatively low velocity, the distribution at the outer collector would be a factor of three broader in the TOF spectra. This is not found, so the important question to answer precisely is then if the signals are due to photons or relativistic particles. As seen in Fig. 4 in the panel named Timing, the signal rise edge is well described by just shifting the signal curves by $3-4 \mathrm{~ns}$, which is the time for relativistic particles to move the $1 \mathrm{~m}$ distance between the two collectors. If the signal was due to photons, the same signal would appear at both collectors but shifted by $4 \mathrm{~ns}$. This is not the case, since some slower part of the signal at the inner collector seems to have vanished before reaching the outer collector. Thus, it is concluded once more that this signal is due to relativistic particles and not to photons.

It is not only that the signal at the outer collector is faster than the signal to the inner collector, but it also often much larger in intensity. Since both collectors are fully illuminated from the target as designed in this experiment, the signal at the outer collector should be decreased by a factor $(64 / 163)^{2}=0.154$ relative to the inner collector in Fig. 4, with the laser impact on the target approximating a point source 
and approximately the same size of the collectors. Such a rescaled TOF intensity is shown in Fig. 4 in the panel named Intensity. It is found to be much smaller than the actual signal observed. This indicates that a large signal intensity has been created in the space between the two collectors, caused by decay of the mesons in the beam which gives more particles with an even higher velocity. These fast particles will give more secondary electrons at the collector and thus a larger observed signal.

\subsection{Meson decay using $D(0)$}

Several well-defined decay time constants are observed in the experiments. As is shown in the theoretical section, the signal will vary with two time constants $\tau_{1}$ and $\tau_{2}$. If further time constants exist, for example by one more step in a linear decay chain, the signal may show further features. Since it is known that the kaon decay is branched (for example, charged kaons form both pions and muons, while pions also form muons), a linear chain (as used here to prove that a decay process exists) is not a complete description. A few other timing effects may also influence the results, namely the laser pulse width, the decay-time of the plasma formed and the TOF transport times. Such effects will be studied below in experiments with large collector bias voltage. Despite this, quite good exponentials and even accurate decay timeconstants are obtained.

One or two well defined decay time-constants are normally observed in all signals independent of the collector bias. In experiments with two relatively short flight distances as those used in Fig. 2, several decay time constants are observed. In Fig. 5, the signal to the inner collector is measured with varying laser intensity. Two time constants are observed, one close to $50 \mathrm{~ns}$, which is close to the decay time constant for the long-lived neutral kaon, and one at 39 ns which is proposed to be a relativistically dilated decay time-constant for charged pions [39]. Even with a relatively short flight distance to the outer collector at $103 \mathrm{~cm}$ as used in Fig. 6 in the same experimental run, the pattern of decays changes considerably relative to the inner collector signal in Fig. 5 due to meson cecay in the beam. The long time constant at $50 \mathrm{~ns}$ is no longer clearly observed, and $39 \mathrm{~ns}$ is an upper limit for the main decay time constant. Two shorter time constants close to 26 and 13 ns appear. Thus, shorter decay time constants are observed at longer distance, which means that the time constants observed are not simply due to a transport times or pulse timing effects at the target. It is apparent that these effects are due to the decay of several types of mesons with different kinetic energies, as shown in a recent publication [39] and summarized in the Appendix.

Similar experiments with a longer flight path of $163 \mathrm{~cm}$ and with variation of the laser intensity (similar to Figs. 5 and 6) are shown in Fig. 7. These results can thus be compared with Fig. 6 at $103 \mathrm{~cm}$. The decay time constants observed are similar, at approximately 13 and $26 \mathrm{~ns}$ and also the dilated constant at $39 \mathrm{~ns}$ [39] is observed (see the Appendix). Another series of experiments in Fig. 8 checked for a target temperature dependence, which was found to be negligible. The signal with negative bias at the outer collector in Fig. 8b clearly has a short 13 ns decay, thus shorter than the simultaneous decay timeconstant at the inner collector at $26 \mathrm{~ns}$ in Fig. 8a. This proves that meson decay has taken place in the beam. These decay time-constants are close to that for charged kaons, which is $12.4 \mathrm{~ns}$, and to that for charged pions at $26.0 \mathrm{~ns}[21,40,41]$. Since kaons decay to pions, both these types of mesons are formed. 
A model explaining how it is possible that shorter time constants are observed at the outer collector is given in Fig. 9. The reason why muons from pions are not observed at the outer collector is that the pions may be formed from neutral kaons with randomly directed kinetic energy, which brings most of the muons from these decaying pions out from the beam. Thus, the general behavior of the signals at the two collectors is understood. Simulations of the signal behavior are unlikely to provide any important information. Further high-precision decay time constants have been published [39]. The setups for such measurements need to be slightly different since only one meson type should be observed in each experiment, but these problems have been solved successfully as seen in the Appendix.

The best decay time constant observed in the present experiments at the inner collector with negative bias in Fig. 10 gives $\tau_{2}=25.2 \pm 0.1 \mathrm{~ns}$ (fit errror). This is $3 \%$ from the accepted decay of charged pions at $26.0 \mathrm{~ns}[21,40,41]$. As can be seen in Fig. 10, a slightly too short decay time is likely to be found here due to the inclusion also of the upper part of the decaying signal which may be caused by the overlapping 13 ns time constant signal from charged kaons (to just remove that part would be incorrect). Another factor may also be the short distance to the collector. Thus, accurate values for meson decay time constants can be found from the present experiments. The full spectrum of meson decay time constants has been observed in other experiments [39] as also summarized in the Appendix. The best fit for the pions given there is $0.4 \%$ from the accepted value, with a fit error of $\pm 0.04 \mathrm{~ns}$.

One further parameter which can be varied in the experiments is the voltage of the collectors. Other effects like $\mathrm{e}^{ \pm}$pair production [12] may then also become apparent, but at relatively low negative voltage bias the behavior agrees with the results above. In Figs. $11 \mathrm{a}$ and $11 \mathrm{~b}$, the signals at the inner and outer collectors are shown at a bias of 0 to $-600 \mathrm{~V}$. The main time constant is found at $13 \mathrm{~ns}$ as in most results above. An even shorter time constant at $7 \mathrm{~ns}$ is observed in both figures at high voltage. This time constant is close to the laser and plasma pulse lengths. This signal is probably due to photoelectrons formed at the collector by gamma photons from the target. These photoelectrons are formed with low kinetic energy and are not ejected rapidly at low voltage, thus giving a weak and slow signal at low extraction voltage. Only at large negative collector voltage are they ejected so rapidly that the true shape of the gamma pulse is observed. The signal shows the short pulse expected for very fast transport from the target. Thus, at large negative bias voltage of the collector the secondary charge signal due to gamma photons from the plasma at the target is observed.

\subsection{Meson decay using $p(0)$}

Ultra-dense protium $p(0)[1,3]$ has also been studied in this type of experiment. Time constants close to 26 and 13 ns in Figs. 12-13 are found similar to the case of $D(0)$. In Fig. 14, an experiment with variation of the laser intensity is shown, which might be comparable to Fig. 5 using $D(0)$, however in the present experiment a much narrower defining slit is used at the opening to the detector chamber giving lower signal intensity. The time constant 26 ns dominates for $\mathrm{p}(0)$ at the inner collector in Fig. 14, and the signal to the outer collector in Fig. 6 resembles Fig. 14 more than Fig. 5 does. The 26 ns time constant also dominates at the outer collector at $103 \mathrm{~cm}$ distance, which is different from the case of $D(0)$. No 
principle change is thus found for $p(0)$, which means that the nuclear processes giving the fast mesons and muons are not fusion processes which hardly exist with $p(0)$ but are annihilation processes $[39,42]$.

\subsection{Energy gain}

The total energy of the ejected and detected particles can be calculated, but this is a complex process since the particles change both their kinetic energy and their mass on their way from the laser target surface to the final impingement on the collector. So where should the signal be measured? The number of electrons ejected from the collector foil per impinging muon is not well known either. Thus, the current signal in the beam is better measured with a current coil [42,46,47], but its absolute calibration is not trivial. The direct thermal measurements in Ref. [14] are of course valid, but the process taking place was at that time still believed to be nuclear fusion. No particles other than neutrons were assumed to penetrate and leave the apparatus. Later studies have shown that mesons and muons were formed [1517] which left the apparatus with little energy deposition, and also that relativistic particles were formed with energy 100 times higher than possible from fusion. Thus it appears that most particles left the apparatus without depositing their kinetic energy or their decay energy thus their mass. It is possible to estimate the energy released in two ways, from the number of particles formed [47] which is up to $2 \times 10^{14}$ per laser pulse of $0.4 \mathrm{~J}$, using 1 ) the typical kinetic energy of the particles ejected, or 2) using the total mass of the particles, if they would be formed by laser energy or some other unknown energy. The total energy was also calculated in the recent Ref. [48].

Starting with the second method above, and assuming that all particles formed are almost stationary kaons, gives $16 \mathrm{~kJ}$, thus an energy gain of approximately 40000 from the laser pulse. This large gain indicates that the particles observed are not created by the laser but formed from the baryons in $H(0)$. Assuming instead that all particles are formed from the baryons in $\mathrm{H}(0)$ and have a kinetic energy of 100 $\mathrm{MeV}$ on average which agrees with experiments [39], the gain from the laser pulse to kinetic energy is approximately 8000 . This large value proves finally that annihilation is the only possible source of kinetic energy for the mesons.

\subsection{Meson production mechanisms}

One important observation is that the signal observed at the outer collector often is much faster than that at the inner collector. However, in Fig. 2 with short distances, the signal at the outer collector at $103 \mathrm{~cm}$ distance agrees with that at the inner collector at $64 \mathrm{~cm}$, giving a nice overlap after rescaling of the distances. This indicates that the same particles are observed at both collectors in this experiment. In experiments where the effects of meson decay clearly dominate like in Fig. 8a and 8b, this is no longer true. There, the peak at long distance in Fig. $8 \mathrm{~b}$ is at $32 \mathrm{~ns}$ or $13 \mathrm{MeV} \mathrm{u}^{-1}$, while that at short distance in Fig. $8 \mathrm{a}$ is at $20 \mathrm{~ns}$ or $5 \mathrm{MeV} \mathrm{u}^{-1}$. This means that the particles observed at the outer collector are much faster than those observed at the inner collector, which is due to the respective decay processes as shown in Fig. 9. This figure was explained above where the signal at the inner collector was concluded to be due to muons formed with relatively low energy from decaying pions, while that at the outer collector was concluded to be due to muons formed with much higher energy from decaying kaons, as given by their 
respective characteristic decay times. It is thus important to understand the different processes taking place in the particle beam moving to the collectors.

It is apparent from the results described above that a process which releases a very high energy is involved in the formation of the mesons. The mechanism investigated here has thus two different parts:

1) the mechanism releasing such a high energy of the order of many $\mathrm{J}$ (as described above total $800 \mathrm{~J}$ from $0.1 \mathrm{~J}$ laser pulse), and 2) the processes forming the mesons. There exists only one possibility for the high energy release and that is baryon annihilation [39, 42], and this is known to be a process which gives meson bunches [49] similar to those found here. The detailed nuclear processes starting the annihilation process are outside the present study. The method of investigation used here gives extremely high meson fluxes, thus this method can replace large accelerators used for meson production, so called meson factories.

The annihilation reaction giving the mesons has been published previously [39]. The energy from a neutron plus antineutron annihilation creates mesons according to

$$
\begin{aligned}
& n+\bar{n} \rightarrow 2 \mathrm{~K}^{0}(96 \mathrm{MeV})+2 \pi^{ \pm}(69 \mathrm{MeV})+2 \pi^{ \pm}(0 \mathrm{MeV}) \\
& 2 \times 939.6-2 \times(497.7+96)-2 \times(139.6+69)-2 \times(139.6)= \\
& 1879.2-1883.8=-4.6 \mathrm{MeV}
\end{aligned}
$$

where the kinetic energies of the created particles in parantheses are calculated from their measured dilated decays [49] (see also the Appendix) and the energetics is correctly accounted for at the few MeV level, with a precision within $0.3 \%$. An anti-proton annihilating a proton gives

$$
\begin{aligned}
& p+\bar{p} \rightarrow 2 \mathrm{~K}^{ \pm}(96 \mathrm{MeV})+2 \pi^{ \pm}(69 \mathrm{MeV})+2 \pi^{ \pm}(0 \mathrm{MeV}) \\
& 2 \times 938-2 \times(493.7+96)-2 \times(139.6+69)-2 \times(139.6)= \\
& 1876-1876=-0 \mathrm{MeV}
\end{aligned}
$$

This is exact, not including the fit uncertainties from the dilation energy measurements which can be found in the Appendix. Charged kaons may replace the neutral kaons and neutral pions may replace charged pions in these annihilation reactions since their energies of creation are similar.

\section{Discussion}

It should maybe first be stressed that the signals observed have peak voltages of $0.5 \mathrm{~V}$ into $50 \Omega$ input to the oscilloscope, at 1-2 m distance from the laser target, or peak currents of $10 \mathrm{~mA}$. The normal noise from the very quiet laser to the collectors is below $1 \mathrm{mV}$, as seen in Fig. 3. No features of the studied signals can be explained by stable particles like protons, deuterons or electrons from the target. Most of the particles ejected from the laser target are neutral as shown by simple high voltage deflection experiments and several different experiments using deflection in strong magnetic fields [15]. Such 
experiments exclude that the signal at the collectors is due to electrons or positrons. Stable charged particles from the target would not generate a larger and faster signal at a larger collector distance as observed here. If the signal at the TOF maximum at the inner collector in Fig. 5 would be due to electrons or positrons directly from the target, the electron (positron) energy would be around $2 \mathrm{keV}$. Electrons or positrons at $2 \mathrm{keV}$ have a cyclotron radius of $3 \mathrm{~m}$ in the geomagnetic field. At the outer collector $(163 \mathrm{~cm}$, in the east direction relative to the target) the deflection is calculated to be $40 \mathrm{~cm}$. Such particles rapidly reach the wall in the $10 \mathrm{~cm}$ diameter TOF tube and are thus not able to reach the collector at all. Thus, light charged particles from the target like electrons and positrons are excluded as the cause of the signal observed. The origin of the mesons was identified as small non-superfluid clusters $\mathrm{H}_{3}(0)$ and $\mathrm{H}_{4}(0)$ [46], based on the study of the transition temperature for $\mathrm{H}(0)$ from a superfluid to a normal fluid [9].

In experiments on laser-induced processes in $\mathrm{D}(0)$, pair production of lepton pairs was shown to exist [12]. The method used to prove $\mathrm{e}^{ \pm}$pair production was to increase the collector bias voltage to demonstrate lack of saturation of the signals. In the present case, the results are slightly different from those in Ref. [12]. In Fig 11, the TOF signal is shown at the inner and outer collectors, both with negative bias up to $-600 \mathrm{~V}$. A saturation (limiting) of the signal exists. This limiting is observed at the very fast signal which peaks at 20-25 ns time. It was shown above from the signal peaking with time constant of 7 $\mathrm{ns}$, that the peak signal is due to secondary electrons formed at the collector, probably photoelectrons from gamma radiation. In such a case, a saturation at high voltage will exist and pair production will not dominate the signal. No saturation of the signal with positive bias is in fact observed in the present experiments which indicates a signal due to pair production, but those results are not presented here since they are not important for the conclusions of the present study.

The transport time of the particles in the beam is of little concern here, since the particles observed have very high velocity during their transport to the collectors. However, such effects may be seen in the matching of Eq. (3) to the meson decay signals. In most cases the matching of the results here includes a shift possibly due to transport and certainly to other broadening effects like the laser and plasma pulse widths. This shift is 11-15 ns for most signals at all collector distances and almost no systematic variation is observed. Only in the fastest case in Fig. 11a and b, there is a clear difference between the shifts at the inner and outer collectors, being $15 \mathrm{~ns}$ for the $64 \mathrm{~cm}$ distance and $19 \mathrm{~ns}$ for the $163 \mathrm{~cm}$ distance. The peak of these signals in Fig. 11 was concluded to be due to photons, and the $4 \mathrm{~ns}$ difference for $99 \mathrm{~cm}$ travel agrees well with the speed of light. In most experiments different muon signals dominate at the inner and outer collectors, mainly due to pions at short distance and kaons at larger distance. This is certainly the reason why the signal at the outer collector is not systematically shifted to slower times relative to the inner collector. For example, in Fig. 5 the first time constant $\tau_{1}$ for conversion from the initially ejected species to the decaying pion is $2 \mathrm{~ns}$. This signal in Fig. 5 is to the collector at $63 \mathrm{~cm}$ distance, while the signal to the outer collector at $103 \mathrm{~cm}$ distance is shown in Fig. 6 , with $\tau_{1}=9 \mathrm{~ns}$. This may indicate a transport time of $7 \mathrm{~ns}$ over $39 \mathrm{~cm}$ distance corresponding to a kinetic energy close to $20 \mathrm{MeV} \mathrm{u}^{-1}$. The most apparent similar effect is observed in the $\tau_{1}$ constant in Figs. 8a and $\mathrm{b}$. In the case of the inner collector with signal from pion decay this constant is $5 \mathrm{~ns}$, while at the 
outer collector with signal from kaon decay in Fig. $8 \mathrm{~b}, \tau_{1}$ is $11 \mathrm{~ns}$. This difference could possibly be due to a transport time of $6 \mathrm{~ns}$ over the $99 \mathrm{~cm}$ distance between the two collectors, corresponding to a kinetic energy of $150 \mathrm{MeV} \mathrm{u}^{-1}$.

The signal variation with varying laser intensity in Figs.5-7 and 14 is in general exponential with time. When the total signal is low, as found with low laser intensity, the signal decays into the noise much faster than at large laser intensity, However, also the time dependence varies. Sometimes this is seen as in Fig. $7 \mathrm{~b}$, where it is very clear that the $13 \mathrm{~ns}$ decay disappears at the lowest laser intensity used. This time constant, which is more apparent at high intensity, is due to $\mathrm{K}^{ \pm}$decay, while the $26 \mathrm{~ns}$ time constant is due to $\pi^{ \pm}$decay. This would indicate that the formation of $\pi^{ \pm}$is independent of the formation of $K^{ \pm}$. Thus $\pi^{ \pm}$is here probably formed from $\mathrm{K}_{s}^{0}$, which appears to be formed at lower laser energy than $\mathrm{K}^{ \pm}$. In Fig. 6, a different situation appears to exist. There the $13 \mathrm{~ns}$ signal from $\mathrm{K}^{ \pm}$exists also at low intensity, while the $26 \mathrm{~ns}$ decay from $\pi^{ \pm}$has disappeared. Thus, in this case it is likely that the pions observed are formed from the $\mathrm{K}^{ \pm}$which remains at low laser intensity while the pions produced by the $\mathrm{K}^{ \pm}$decay at low laser intensity are ejected in random directions and give too low signal at the outer collector to be observed.

One point to discuss is if other mesons are involved in the formation of the observed kaons and pions. One possibility for this is various $D$ mesons. Their energy is close to two baryons so $D$ mesons might be formed by an initial reaction between a neutron and an antineutron (or similar) before decay to kaons and pions. Due to their short lifetimes ( 1 ps or less) it is unlikely that any direct evidence can be found in the present experiments, and the experiments are not better understood from any intermediate formation of $D$ mesons. However, the oscillation between matter and antimatter forms recently reported [51] is similar to the oscillations which take place in the present experiments [39,42].

Another point of interest is what particles that finally leave the chamber. Due to their short lifetimes, the kaons and pions will decay to form muons $\mu^{ \pm}$inside the chamber The muon lifetime is $2.20 \mu$ s, which means that the muons will pass out through the apparatus walls or may be captured if the muons are negative [45]. In the end, they either decay to other leptons or react with heavy nuclei to form relatively shortlived isotopes [45].

\section{Conclusions}

It is shown that the particles ejected from the laser-induced processes in $\mathrm{H}(0)$ have a short life-time, and that they often decay to charged particles at a short distance from the laser target. The signals measured at three different distances agree with the theoretical time variation of an intermediate meson signal, mainly displaying time constants close to 13 and $26 \mathrm{~ns}$. These values are the time constants for decay of charged kaons and pions. A large energy gain is observed, with the kinetic energy of the particles at least 8000 times larger than the laser pulse energy, thus at $800 \mathrm{~J}$. Due to the large number of different mesons formed, fits to the final time-dependent signals with several parameters, one for each meson type, are 
meaningless. Instead, the present paper reports on the physics of the meson formation and decay processes, while more exact life-times have been published [39] (with precision better than $1 \%$ for all types of mesons with lifetime in the ns range) (see also the Appendix below).

\section{References}

[1] L. Holmlid and Sindre Zeiner-Gundersen, "Ultradense protium $\mathrm{p}(0)$ and deuterium $\mathrm{D}(0)$ and their relation to ordinary matter: a review". Physica Scripta 74 (7) 2019.

https://doi.org/10.1088/1402-4896/ab1276.

[2] L. Holmlid, "Excitation levels in ultra-dense hydrogen $\mathrm{p}(-1)$ and $\mathrm{d}(-1)$ clusters: structure of spin-based Rydberg Matter". Int. J. Mass Spectrom. 352 (2013) 1- 8. DOI: 10.1016/j.jjms.2013.08.003.

[3] L. Holmlid, "Laser-mass spectrometry study of ultra-dense protium $\mathrm{p}(-1)$ with variable time-of-flight energy and flight length".

Int. J. Mass Spectrom. 351 (2013) 61-68. DOI: 10.1016/j.jjms.2013.04.006

[4] L. Holmlid, "Emission spectroscopy of IR laser-induced processes in ultra-dense deuterium D(0): Rotational transitions with spin values $s=2,3$ and 4 ".

J. Mol. Struct. 1130 (2017) 829-836.Doi: 10.1016/j.molstruc.2016.10.091.

[5] L. Holmlid, "Rotational emission spectroscopy in ultra-dense hydrogen $\mathrm{p}(0)$ and $\mathrm{pxDy}(0)$ : groups $\mathrm{pN}$, pD2, p2D and (pD) $)_{N}$. Journal of Molecular Structure 1173, 567-573 (2018) https://doi.org/10.1016/j.molstruc.2018.06.116

[6] P.U. Andersson, B Lönn and L. Holmlid, "Efficient source for the production of ultra-dense deuterium $\mathrm{D}(-1)$ for laser-induced fusion (ICF)".

Rev. Sci. Instrum. 82 (2011) 013503. doi:10.1063/1.3514985.

[7] P.U. Andersson and L. Holmlid, "Superfluid ultra-dense deuterium D(-1) at room temperature". Phys. Lett. A 375 (2011) 1344-1347. doi:10.1016/j.physleta.2011.01.035.

[8] P.U. Andersson, L. Holmlid, and S.R. Fuelling, "Search for superconductivity in ultra-dense deuterium $D(-1)$ at room temperature: depletion of $D(-1)$ at field strength $>0.05 \mathrm{~T}$ ".

J. Supercond. Novel Magn. 25 (2012) 873-882.

DOI: $10.1007 / \mathrm{s} 10948-011-1371-6$.

[9] L. Holmlid and B. Kotzias, "Phase transition temperatures of 405-725 K in superfluid ultra-dense hydrogen clusters on metal surfaces". 
AlP Advances 6 (2016) 045111. doi: 10.1063/1.4947276.

[10] L. Holmlid, A. Kotarba and P. Stelmachowski, “Production of ultra-dense hydrogen H(0): A novel nuclear fuel", International Journal of Hydrogen Energy 46 (2021) 18466-18480, https://doi.org/10.1016/j.ijhydene.2021.02.221.

[11] L. L. Holmlid, "Two-collector timing of 3-14 MeV/u particles from laser-induced processes in ultradense deuterium".

Int. J. Modern Phys. E 22 (2013) 1350089. DOI: 10.1142/S0218301313500894

[12] F. Olofson and L. Holmlid, "Electron-positron pair production observed from laser-induced processes in ultra-dense deuterium $\mathrm{D}(-1)^{\prime \prime}$.

Laser Part. Beams 32 (2014) 537-548. doi:10.1017/S0263034614000494.

[13] F. Olofson and L. Holmlid, "Time-of-flight of He ions from laser-induced processes in ultra-dense deuterium $\mathrm{D}(0)$ ".

Int. J. Mass Spectrom. 374 (2014) 33-38. DOI: 10.1016/j.ijms.2014.10.004.

[14] L. Holmlid, "Heat generation above break-even from laser-induced fusion in ultra-dense deuterium". AlP Advances 5 (2015) 087129. doi: 10.1063/1.4928572.

[15] L. Holmlid, "Mesons from laser-induced processes in ultra-dense hydrogen $\mathrm{H}(0)$ ".

PLOS ONE 12 (2017) e0169895. doi:10.1371/journal.Pone.0169895. Retracted by the journal but on thr web. Original version exists at https://gup.ub.gu.se/file/208005.

[16] L. Holmlid, "MeV particles in a decay chain process from laser-induced processes in ultra-dense deuterium D(0)”. Int. J. Modern Phys. E 24 (2015) 1550026. DOI: 10.1142/S0218301315500263

[17] L. Holmlid,“Nuclear particle decay in a multi-MeV beam ejected by pulsed-laser impact on ultra-dense hydrogen H(0)". Int. J. Modern Phys. E 24 (2015) 1550080. DOI: 10.1142/S0218301315500809.

[18] L. Holmlid and S. Olafsson, “Charged particle energy spectra from laser-induced processes: nuclear fusion in ultra-dense deuterium $\mathrm{D}(0)$ ".

Int. J. Hydrogen Energy 41 (2016) 1080-1088. doi:10.1016/j.ijhydene.2015.10.072.

[19] L. Holmlid and S. Olafsson, "Muon detection studied by pulse-height energy analysis: novel converter arrangements". Rev. Sci. Instrum. 86, 083306 (2015). DOI: 10.1063/1.4928109.

[20] L. Holmlid and S. Olafsson, "Decay of muons generated by laser-induced processes in ultra-dense hydrogen”. Heliyon 5 (6) (2019) e01864. Doi: 10.1016/j.heliyon.2019.e01864. 
[21] W. E. Burcham and M. Jobes, "Nuclear and Particle Physics", Pearson, Harlow 1995.

[22] J. Nuckolls, L. Wood, A. Thiessen, and G. Zimmerman, Nature 239, 139-142 (1972).

[23] O. A. Hurricane, D. A. Callahan, D. T. Casey, P. M. Celliers, C. Cerjan, E. L. Dewald,

T. R. Dittrich, T. Döppner, D. E. Hinkel, L. F. Berzak Hopkins, J. L. Kline, S. Le Pape, T. Ma, A. G. MacPhee, J. L. Milovich, A. Pak, H.-S. Park, P. K. Patel, B. A. Remington, J. D. Salmonson, P. T. Springer, and R. Tommasini, Nature 506, 343-348 (2014).

[24] V. A. Smalyuk, Phys. Scr. 86, 058204 (2012).

[25] L. Holmlid, "Ultra-dense hydrogen $\mathrm{H}(-1)$ as the cause of instabilities in laser compression-based nuclear fusion”. J. Fusion Energy 33 (2014) 348-350. DOI: 10.1007/s10894-014-9681-x.

[26] R. Betti, A. A. Solodov, and J. A. Delettrez, C. Zhou, Phys. Plasmas 13, 100703-1-100703-4 (2006).

[27] T. Ditmire, J. Zweiback, V. P. Yanovsky, T. E. Cowan, G. Hays, and K. B. Wharton, Nature 398, 489-492 (1999).

[28] M. Barbui, Woosuk Bang, A. Bonasera, K. Hagel, K. Schmidt, J. Natowitz, G. Giuliani, M. Barbarino, G. Dyer, H. Quevedo, E. Gaul, T. Borger, A. Bernstein, M. Martinez, M. Donovan, T. Ditmire, S. Kimura, M. Mazzocco, F. Consoli, R. De Angelis, and P. Andreoli, J. Phys: Conf. Ser. 420, 012060 (2013).

[29] D. Hilscher, O. Berndt, M. Enke, U. Jahnke, P. V. Nickles, H. Ruhl, and W. Sandner, Phys. Rev. E 64, 016414/1-9 (2001).

[30] M. Gerbaux, M. M. Aleonard, G. Claverie, F. Gobet, F. Hannachi, G. Malka, J. N. Scheurer, M. Tarisien, V. Meot, P. Morel, J. Faure, Y. Glinec, A. Guemnie-Tafo, V. Malka, M. Manclossi, and J. Santos, J. Physique IV (Proceedings) 133, 1139-41 (2006).

[31] I. V. Pogorelsky, M. N. Polyanskiy, M. Babzien, V. Yakimenko, N. P. Dover, C. A. J. Palmer, Z. Najmudin, J. Schreiber, P. Shkolnikov, and G. Dudnikova, Laser Physics 21, 1288-94 (2011).

[32] A. G. Lipson, A. S. Roussetski, A. Takahashi, and J. Kasagi, Bull. Lebedev Phys. Inst. (10) 18-24 (2001).

[33] M. Niiyama, EPJ Web Conf. 37, 01006 (2012).

[34] F. Bellemann, A. Berg, J. Bisplinghoff, G. Bohlscheid, J. Ernst, C. Henrich, F. Hinterberger, R. Ibald, R. Jahn, R. Joosten, K. Kilian, A. Kozela, H. Machner, A. Magiera, J. Munkel, P. von Neumann-Cosel, P. von Rossen, H. Schnitker, K. Scho, J. Smyrski, R. Tolle, and C. Wilkin, Phys. Rev. C 75, 15204-1-8 (2007).

[35] H. Hora and D. H. H. Hoffmann, Laser Part. Beams 26, 503-505 (2008). 
[36] L. Holmlid, “High-charge Coulomb explosions of clusters in ultra-dense deuterium D(-1)". Int. J. Mass Spectrom. 304 (2011) 51-56.doi: 10.1016/j.jjms.2011.04.001.

[37] L. Holmlid and S. Olafsson, "Spontaneous ejection of high-energy particles from ultra-dense deuterium D(0)”. Int. J. Hydr. Energy 40 (2015) 10559-10567. DOI: 10.1016/j.ijhydene.2015.06.116.

[38] Balin DV, Ganzha VA, Kozlov SM, Maev EM, Petrov GE, Soroka MA, Schapkin GN, Semenchuk GG, Trofimov VA, Vasiliev AA, Vorobyov AA, Voropaev NI, Petitjean C, Gartner B, Lauss B, Marton J, Zmeskalc J, Case T, Crowe KM, Kammel P, Hartmann FJ, Faifman MP. Phys. Part. Nuclei 42, 185-214 (2011).

[39] L. Holmlid,"Energy production by laser-induced annihilation in ultradense hydrogen $\mathrm{H}(0)$ ". Int. J. Hydrogen Energy (2021) https://doi.org/10.1016/j.ijhydene.2021.01.212.

[40] A. Kamal, "Particle Physics", Springer-Verlag, Berlin Heidelberg 2014.

[41] Particle Data Group, M. Tanabashi et al. (Particle Data Group), Phys. Rev. D 98, 030001 (2018) and 2019 update. (URL: http://pdg.lbl.gov).

[42] L. Holmlid and S. Olafsson, "Laser-induced annihilation: relativistic particles from ultra-dense hydrogen H(0)". High Energy Density Physics 40 (2021) 100942. https://doi.org/10.1016/j.hedp.2021.100942.

[43] G. R. Meima and P. G. Menon, Appl. Catal. A 212, 239 (2001).

[44] M. Muhler, R. Schlögl, and G. Ertl, J. Catal. 138, 413 (1992).

[45] D. F. Measday, Phys. Rep. 354, 243-409 (2001).

[46]. L. Holmlid, "Laser-induced nuclear processes in ultra-dense hydrogen take place in small nonsuperfluid $\mathrm{H}_{\mathrm{N}}(0)$ clusters". Journal of Cluster Science, 30(1), 235-242. DOI: 10.1007/s10876-018-1480-5.

[47]. L. Holmlid,"Existing source for muon-catalyzed nuclear fusion can give MW thermal fusion generator". Fusion Science and Technology, 75:3, 208-217, DOI: 10.1080/15361055.2018.1546090.

[48]. L. Holmlid and S. Zeiner-Gundersen, "Future interstellar rockets may use laser-induced annihilation reactions for relativistic drive". Acta Astronautica 175 (2020) 32-36. DOI:

10.1016/j.actaastro.2020.05.034.

[49]. E. Klempt, C. Batty, J.-M. Richard, The antinucleon-nucleon interaction at low energy:Annihilation dynamics, Physics Reports 413 (2005) 197-317, doi:10.1016/j.physrep.2005.03.002

[50] L. Holmlid, "Leptons from decay of mesons in the laser-induced particle pulse from ultra-dense protium $\mathrm{p}(0)$ ".

Int. J. Modern Phys. E 25 (2016) 1650085. DOI: 10.1142/S0218301316500853. 
[51]. LHCb collaboration (several hundred names). CERN, Observation of the mass difference between neutral charm-meson eigenstates, arXiv:2106.03744v1 [hep-ex] 7 Jun 2021.

\section{Figures}

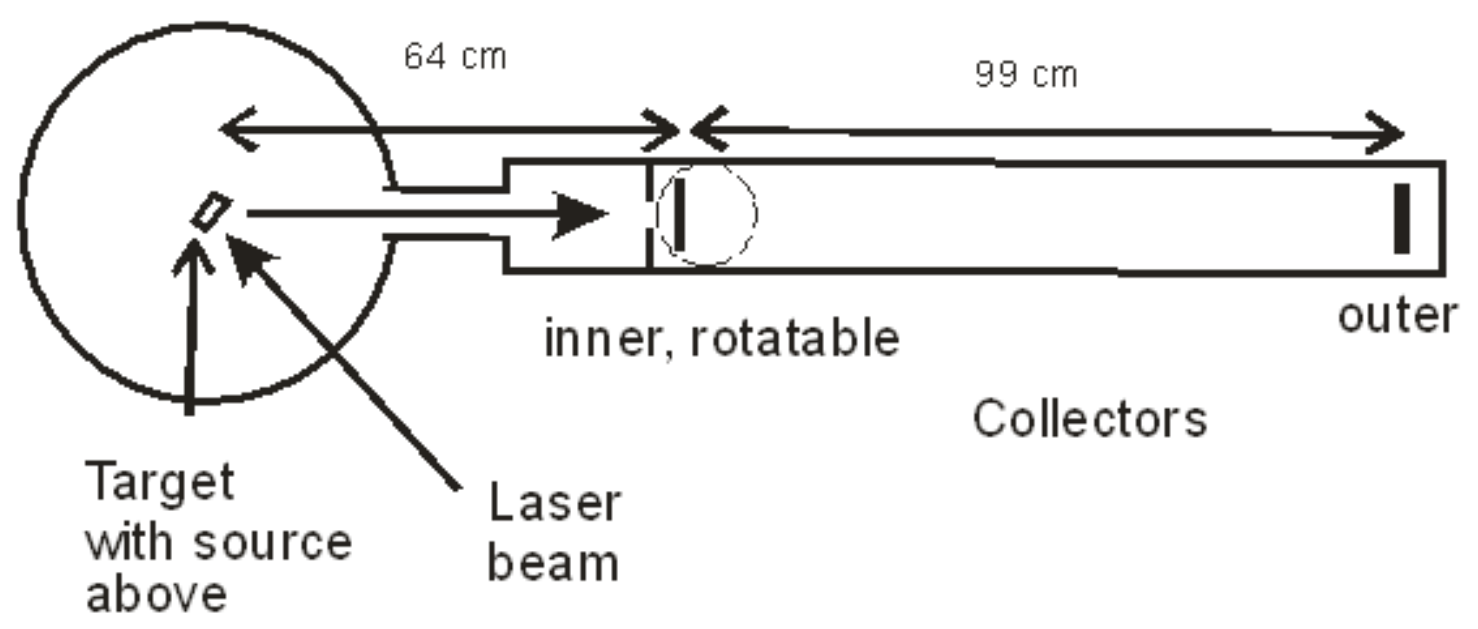

Figure 1

Horizontal cut through the apparatus, not to scale. The particles from the laser target reach the inner collector at a distance of $64 \mathrm{~cm}$ and the outer collector at 163 (shown) or $103 \mathrm{~cm}$. The inner collector is mounted behind an annular plate which covers the opening in the tube. The inner collector can be rotated out of the beam. 

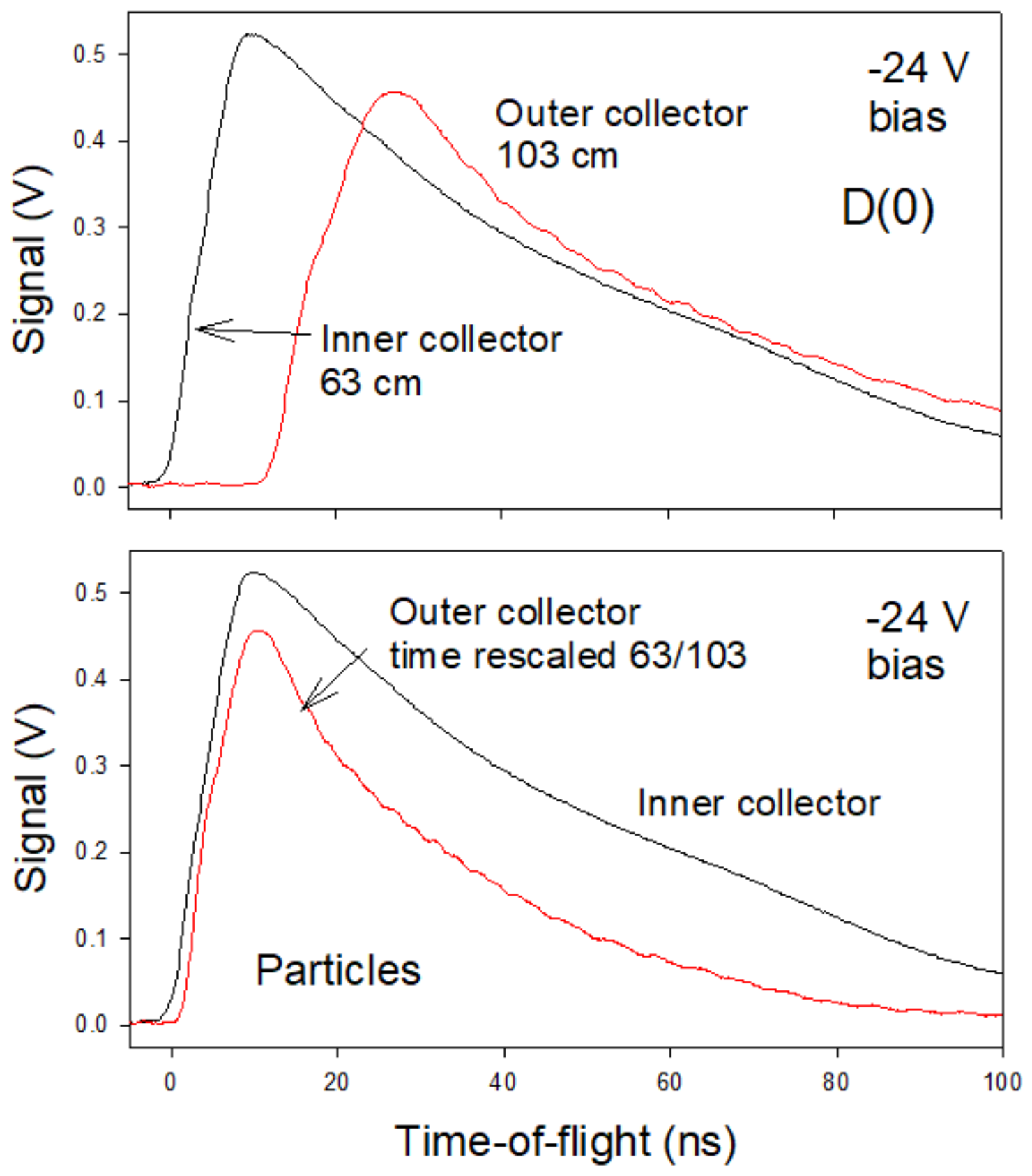

Figure 2

Collector signals with $-24 \mathrm{~V}$ bias for $\mathrm{D}(0)$. The inner collector was open for the outer collector measurement. The outer collector $($ at $103 \mathrm{~cm})$ signal is recalculated to the distance $(63 \mathrm{~cm})$ of the inner collector in the lower panel. The approximate agreement shows that the signal is due to particles with mass and a distribution of velocities. 

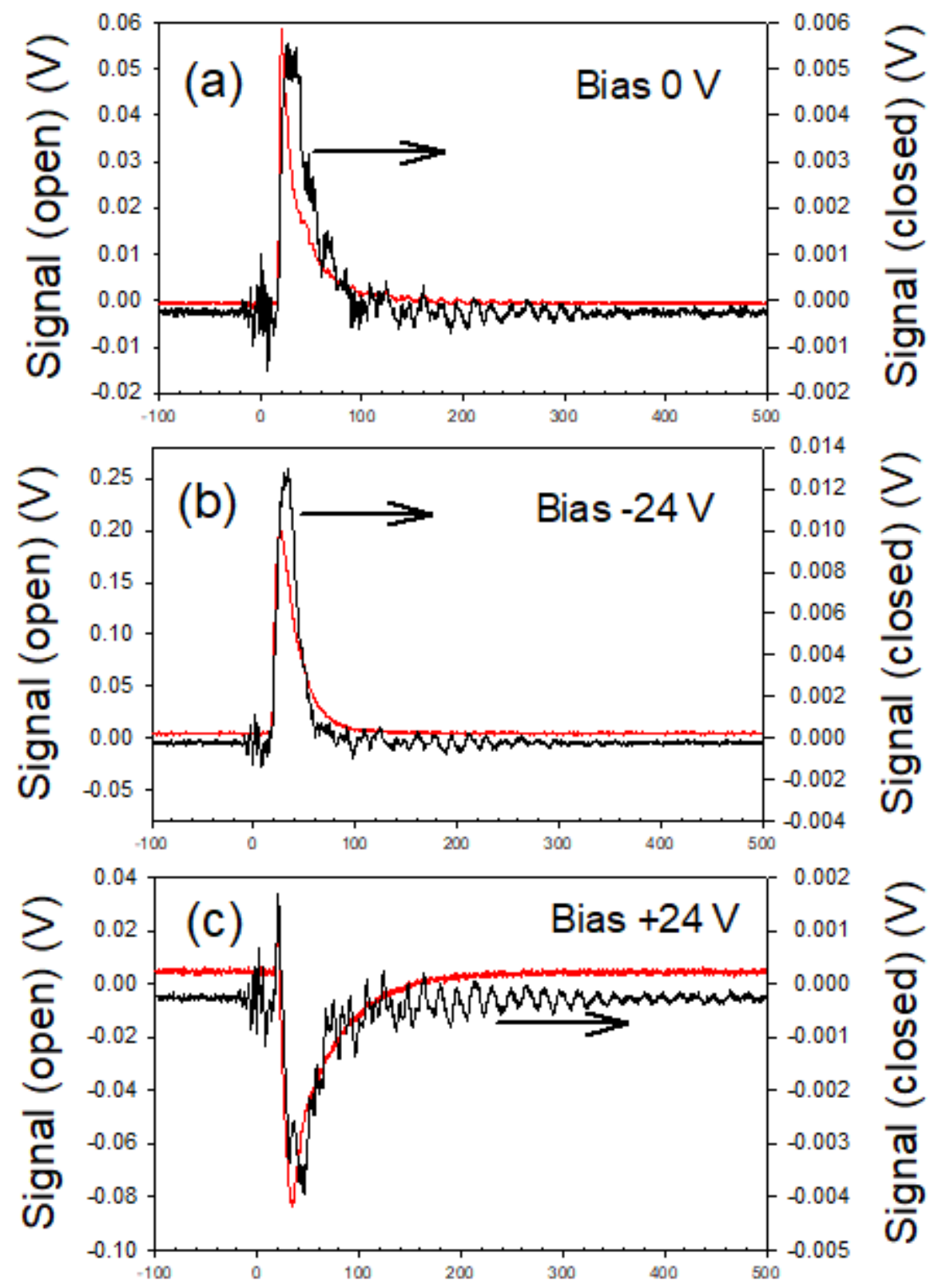

Time-of-flight (ns)

\section{Figure 3}

Signal with $\mathrm{D}(0)$ to the outer collector at zero bias and $163 \mathrm{~cm}$, with the inner collector at $64 \mathrm{~cm}$ open (red) and closed (black). Note that two vertical axes are used, with the signal with the inner collector closed referring to the right-hand axis (shown by the arrow). With closed inner collector, the particles penetrate through the inner collector $(60 \mu \mathrm{m} \mathrm{Al})$ with an attenuation of a factor of 10 (at bias 0 ) or 20 (at bias -24 and $+24 \mathrm{~V}$ ) at $1 \mathrm{~m}$ distance. Thus $5-10 \%$ are transmitted. Note the Compton peak at $+24 \mathrm{~V}$ bias. 


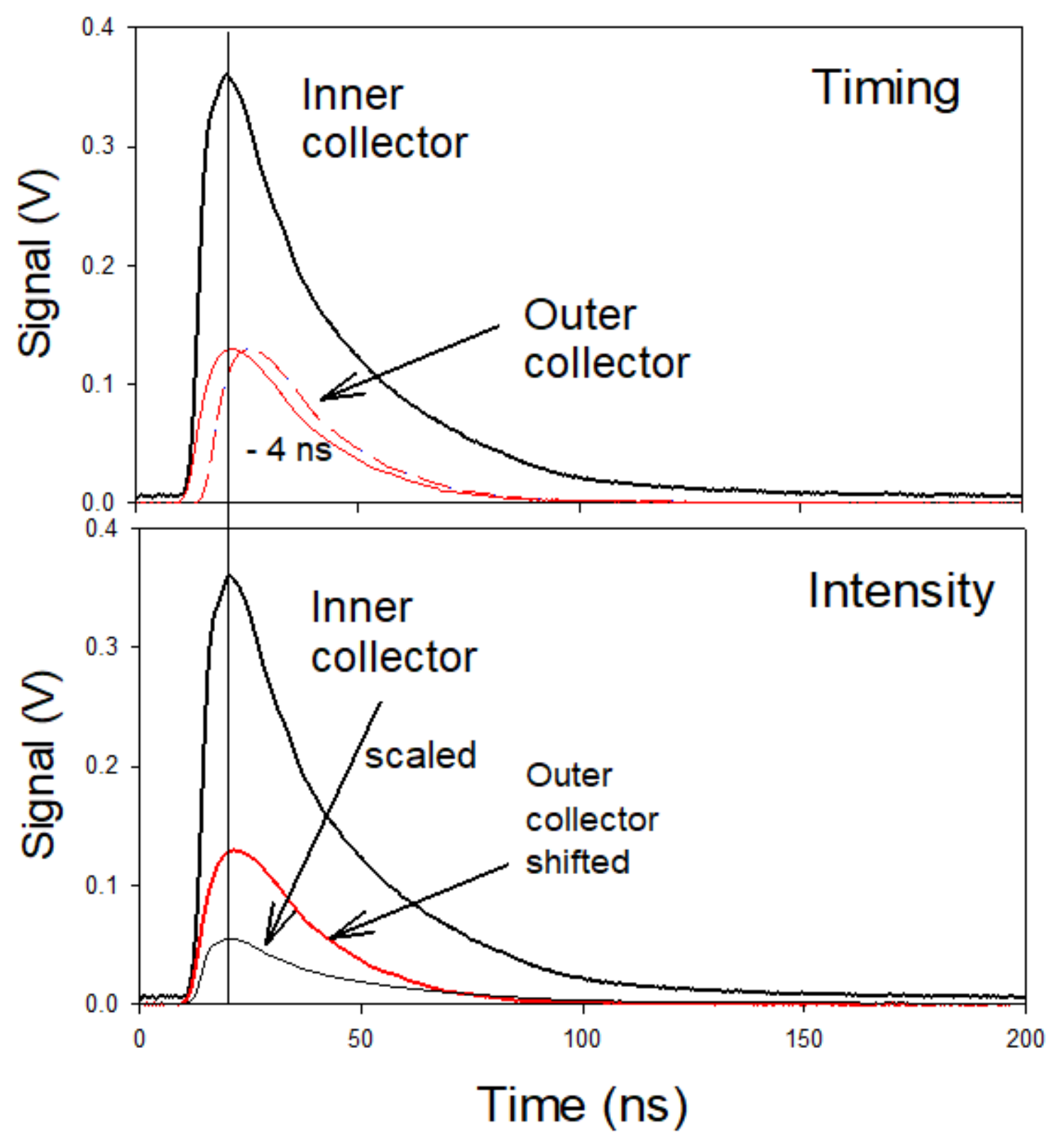

Figure 4

Signals from $D(0)$ to collectors at 64 and $163 \mathrm{~cm}$ distance from the target. Negative bias. In the panel named Timing, the first part of both signals agree after a shift of $4 \mathrm{~ns}$ corresponding to the velocity of light, while the outer collector signal decays much faster. In the panel named Intensity, the outer collector is shown to receive a larger signal than expected from the signal to the inner collector. "Scaled" indicates that the collector signal intensity at $64 \mathrm{~cm}$ is scaled to the outer collector location at $163 \mathrm{~cm}$ by multiplying with (64/163)2. It is seen that the scaled signal is much smaller than the actual signal at 163 $\mathrm{cm}$, so the actual signal intensity is much larger at the outer collector at $163 \mathrm{~cm}$. 


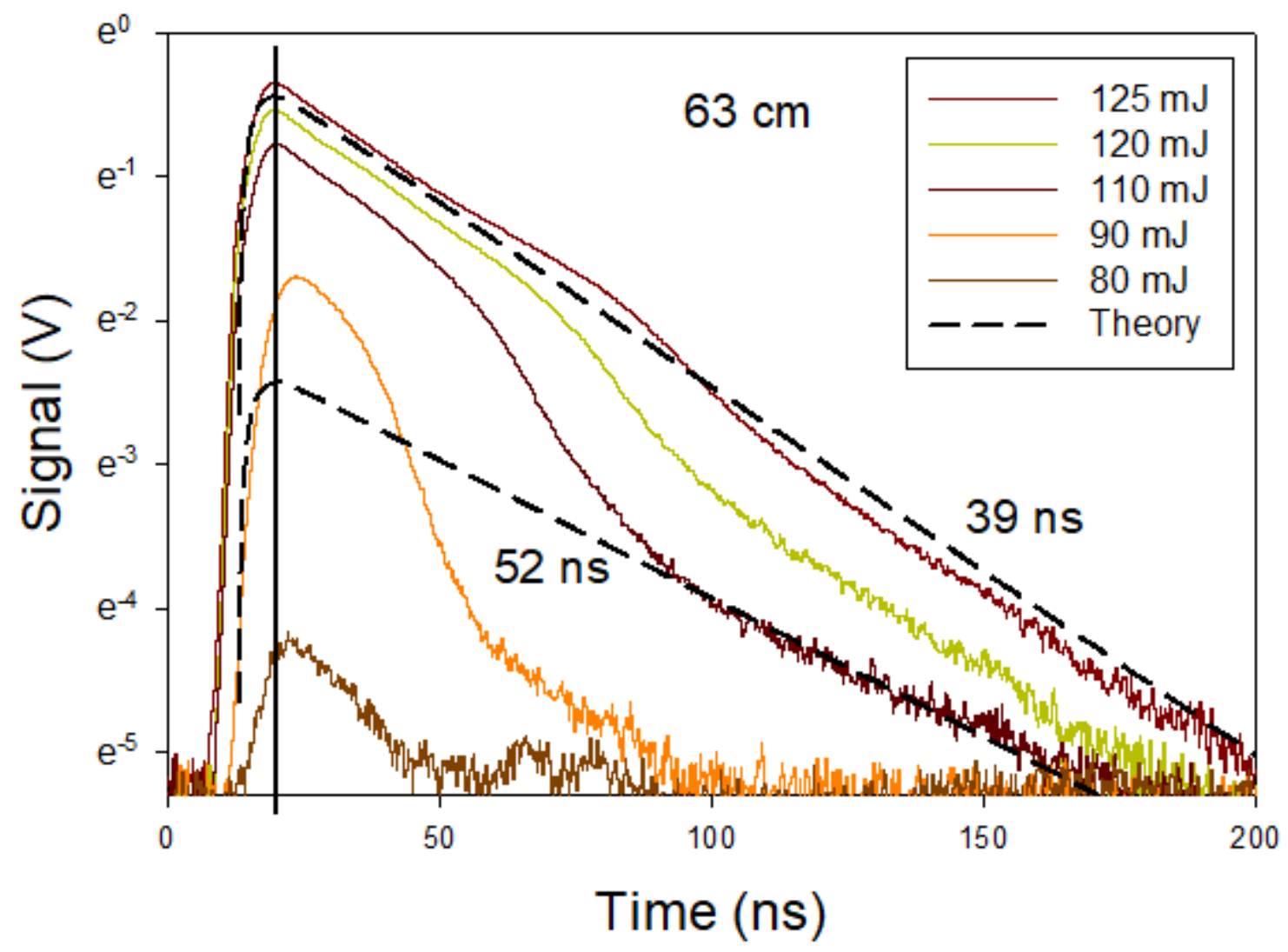

Figure 5

Signal from $\mathrm{D}(0)$ to inner collector at $64 \mathrm{~cm}$ with bias $-24 \mathrm{~V}$. The laser pulse-energy is varied as shown. The dashed curves are calculated. Upper curve $\tau 1=2 \mathrm{~ns}$ and $\tau 2=39 \mathrm{~ns}$, and lower curve $\tau 1=2 \mathrm{~ns}$ and $\tau 2$ $=52 \mathrm{~ns}$. 


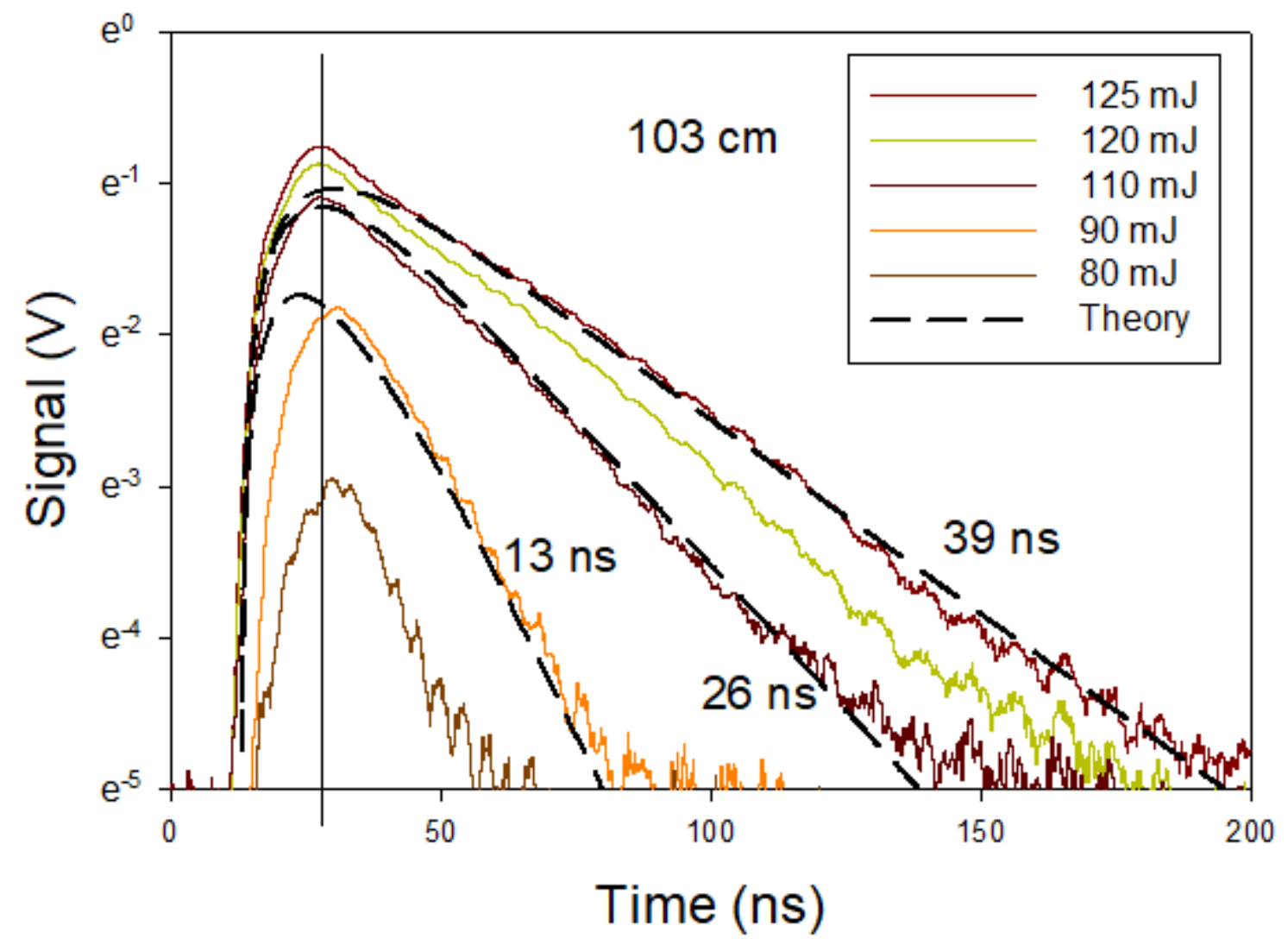

Figure 6

Signal from $\mathrm{D}(0)$ to outer collector at $103 \mathrm{~cm}$ with bias $-24 \mathrm{~V}$. The laser pulse-energy is varied as shown. The dashed curves are calculated. Topmost curve $\tau 1=9 \mathrm{~ns}$ and $\tau 2=39 \mathrm{~ns}$, middle curve $\tau 1=9 \mathrm{~ns}$ and $\tau 2$ $=26 \mathrm{~ns}$, and lower curve $\tau 1=9 \mathrm{~ns}$ and $\tau 2=13 \mathrm{~ns}$. 


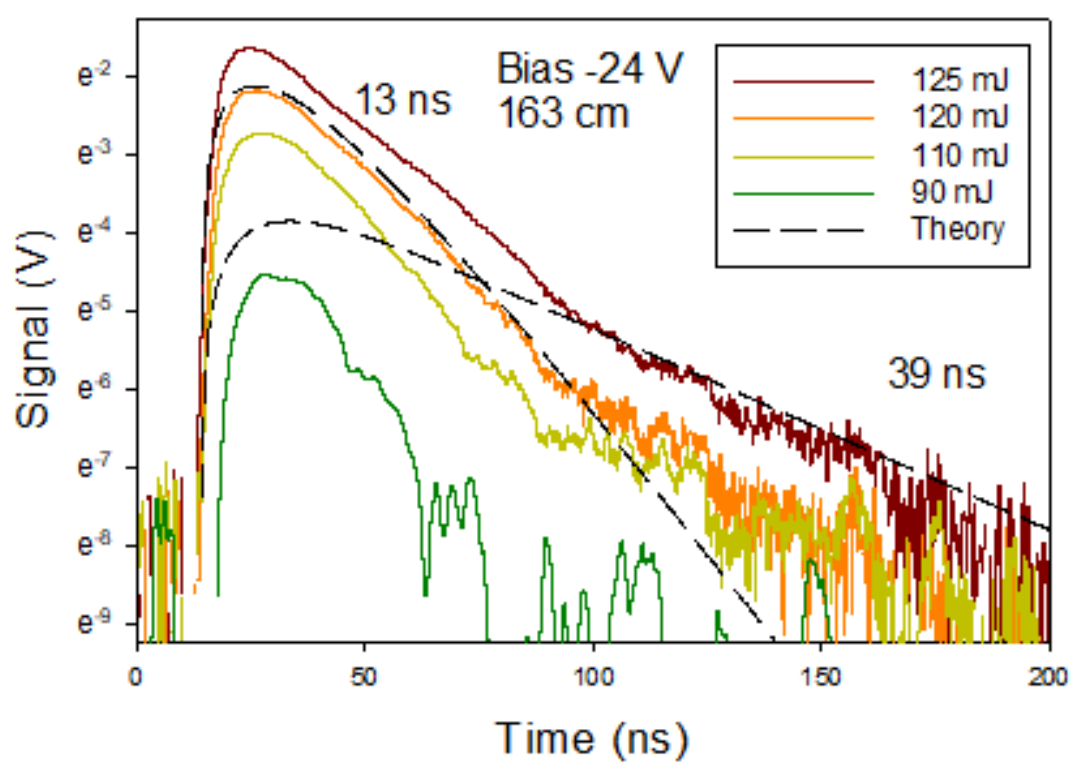

A

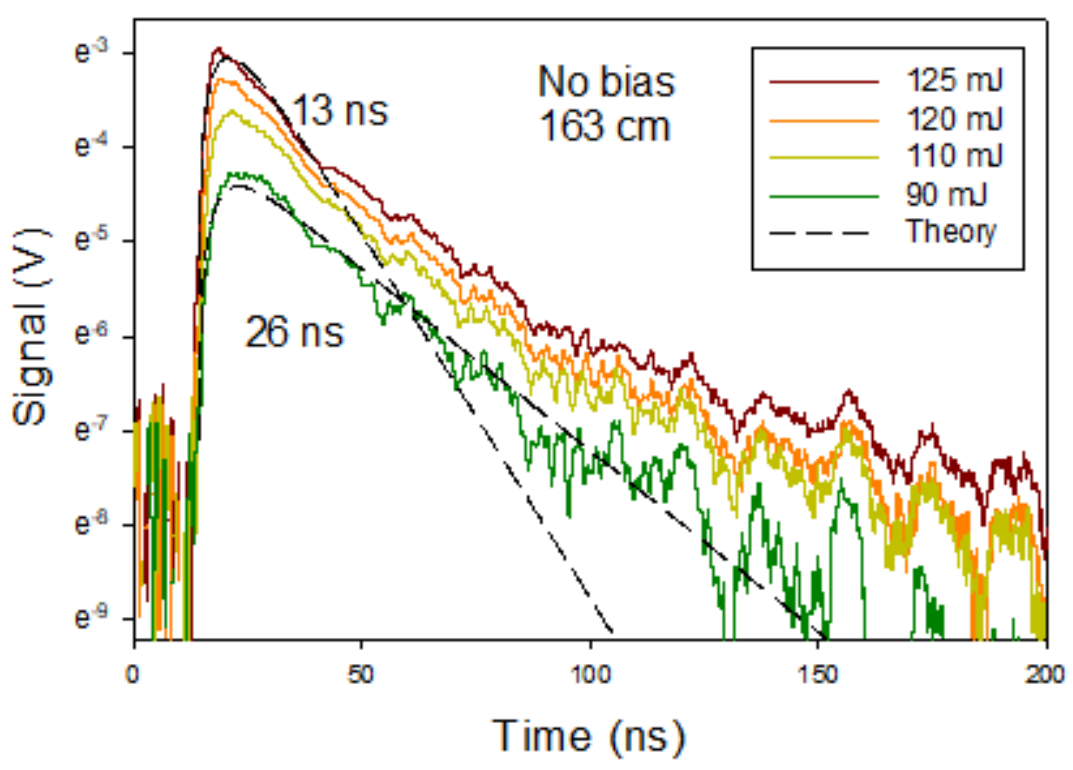

B

\section{Figure 7}

a. Signal from $\mathrm{D}(0)$ to outer collector at $163 \mathrm{~cm}$ with bias $-24 \mathrm{~V}$. Laser pulse-energy is varied as shown. Dashed curves are calculated. Upper curve $\tau 1=11 \mathrm{~ns}$ and $\tau 2=39 \mathrm{~ns}$, and lower curve $\tau 1=11 \mathrm{~ns}$ and $\tau 2$ $=13 \mathrm{~ns}$. b. Signal from $\mathrm{D}(0)$ to outer collector at $163 \mathrm{~cm}$ with no bias $(0 \mathrm{~V})$. Laser pulse-energy is varied as shown. Dashed curves are calculated. Upper curve $\tau 1=4 \mathrm{~ns}$ and $\tau 2=13 \mathrm{~ns}$, and lower curve $\tau 1=4 \mathrm{~ns}$ and $\mathrm{\tau} 2=26 \mathrm{~ns}$ 


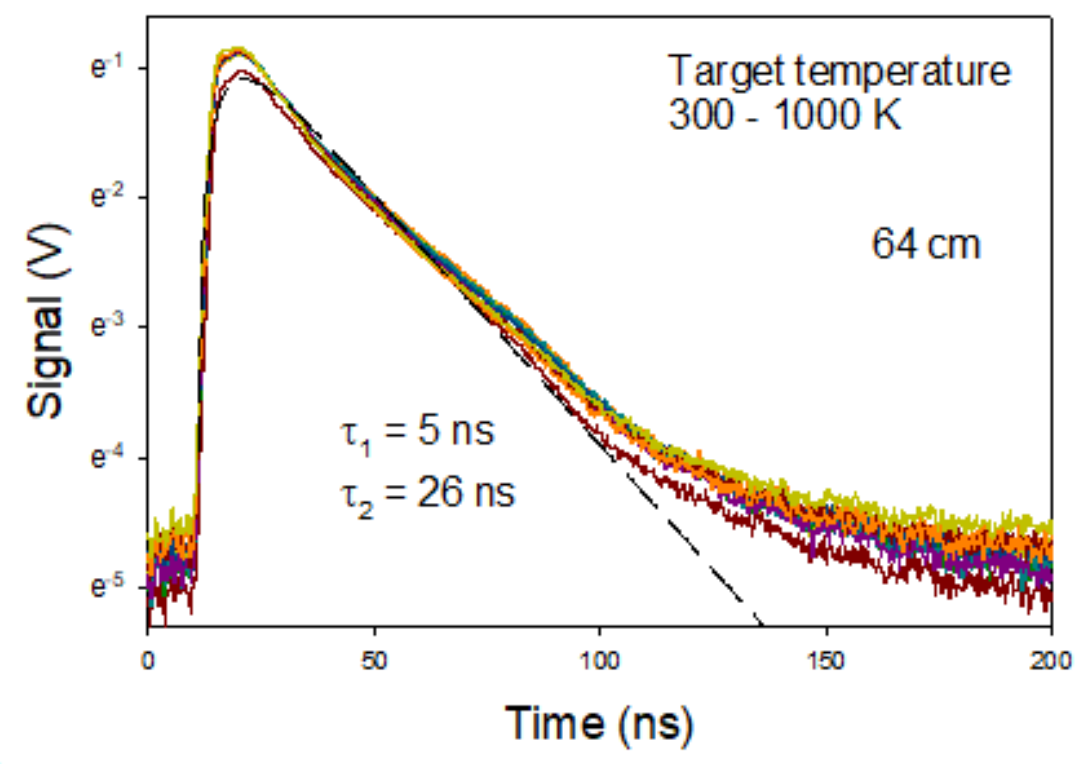

A

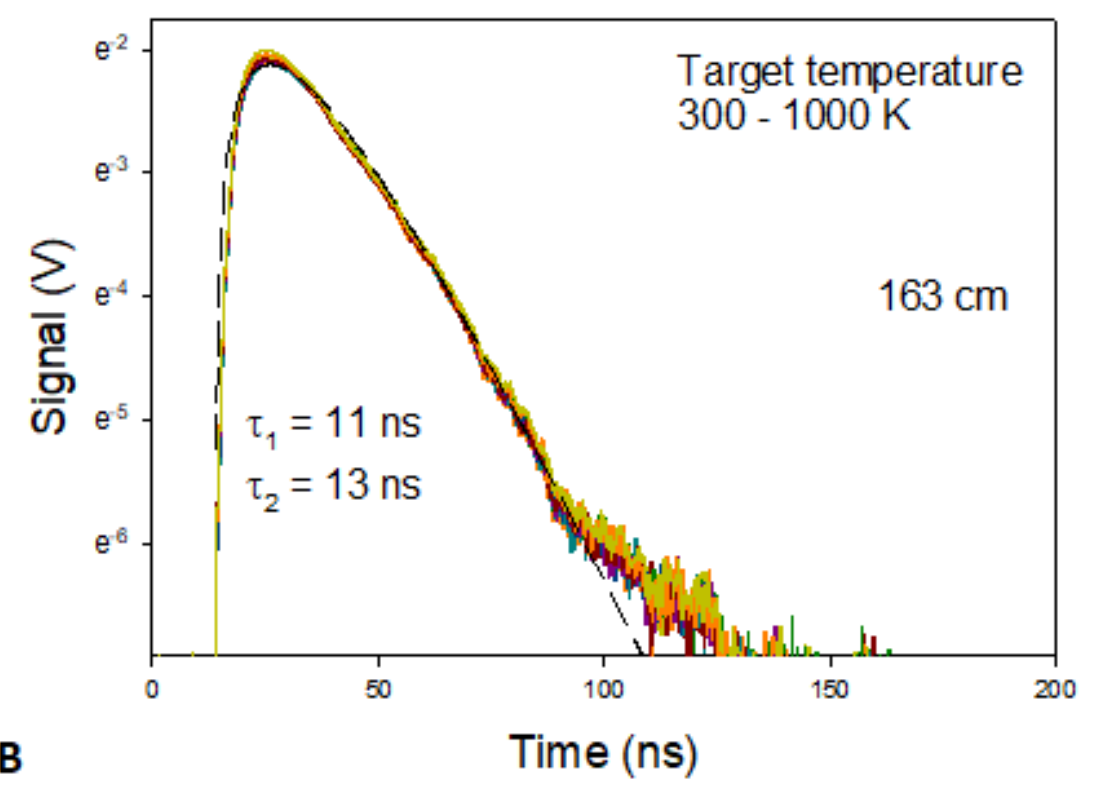

Figure 8

a. Signal from $\mathrm{D}(0)$ to inner collector at $64 \mathrm{~cm}$ with bias $-24 \mathrm{~V}$. Target temperature is varied as shown. Dashed curve is calculated. b. Signal from $D(0)$ to outer collector at $163 \mathrm{~cm}$ with bias $-24 \mathrm{~V}$. Target temperature is varied as shown. Dashed curve is calculated. 


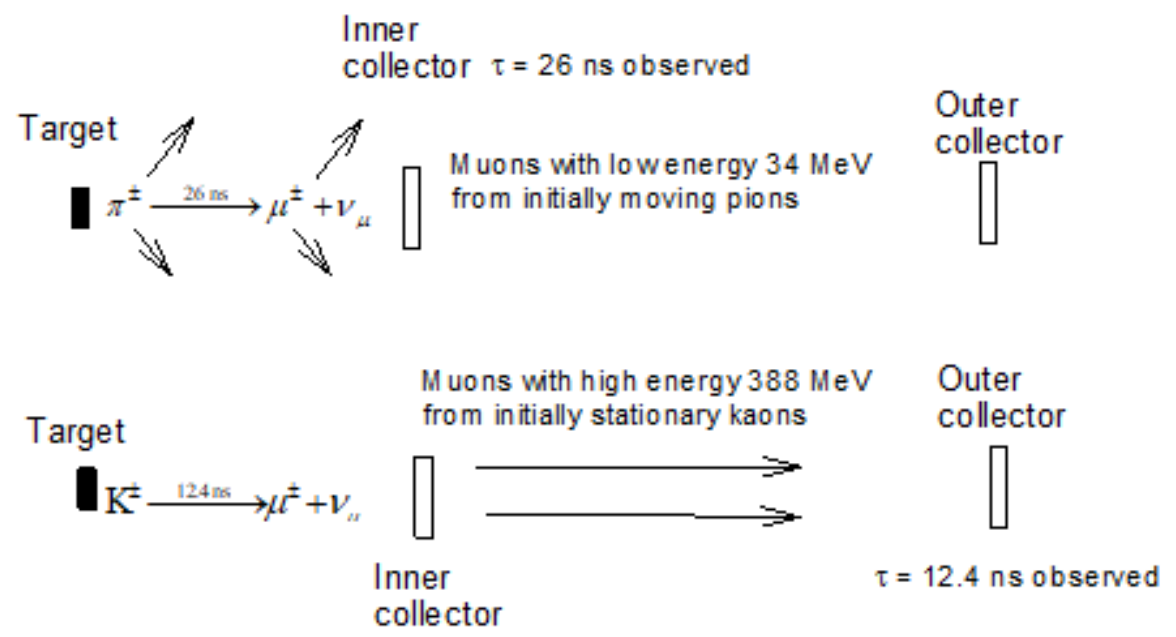

\section{Figure 9}

Model of the experiment: charged kaons $\mathrm{K} \pm$ and charged pions $\pi \pm$ give the two main observed decay time constants. Pions are formed by decay close to the target with excess energy due to their smaller mass, while kaons are more stationary. The signal detected at the collectors is mainly due to muons.

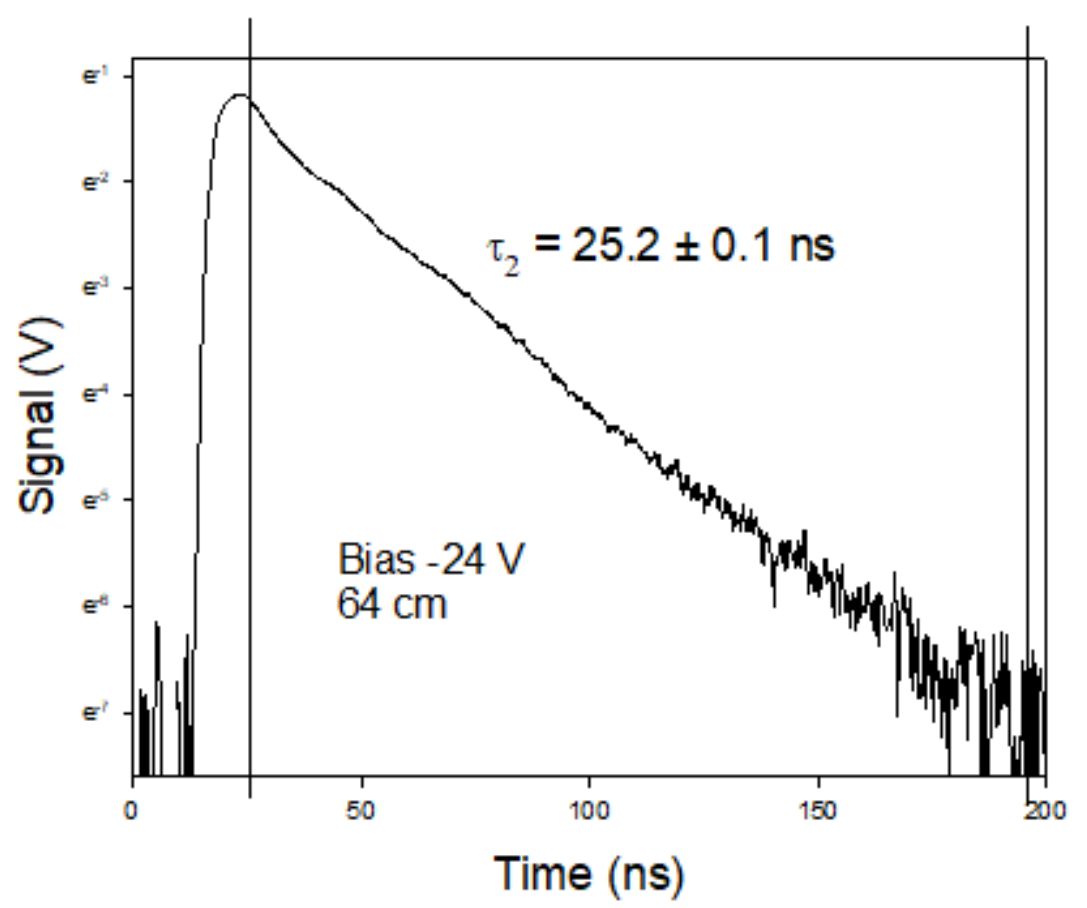

\section{Figure 10}

Signal from $\mathrm{D}(0)$ with $-24 \mathrm{~V}$ bias to the inner collector. The slope between the two vertical lines $(>2$ decades) gives a decay time-constant of $25.2 \pm 0.1 \mathrm{~ns}$. This is close to the time-constant for charged pions of $26.0 \mathrm{~ns}$. 


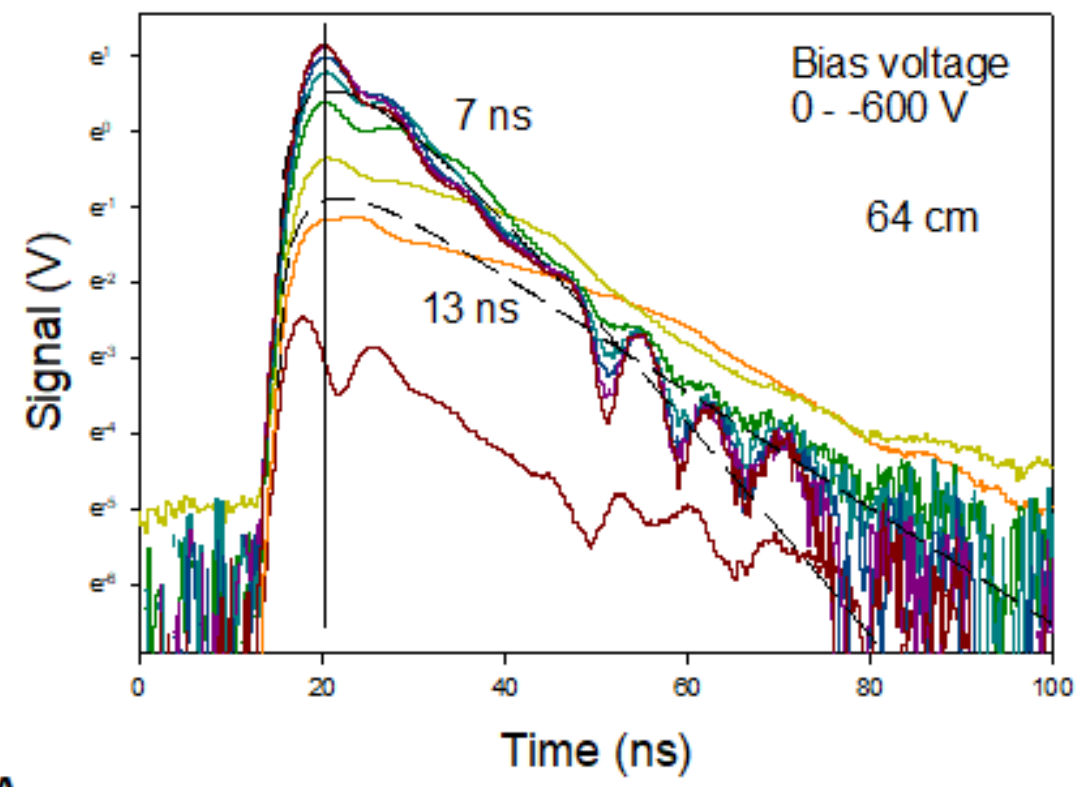

A

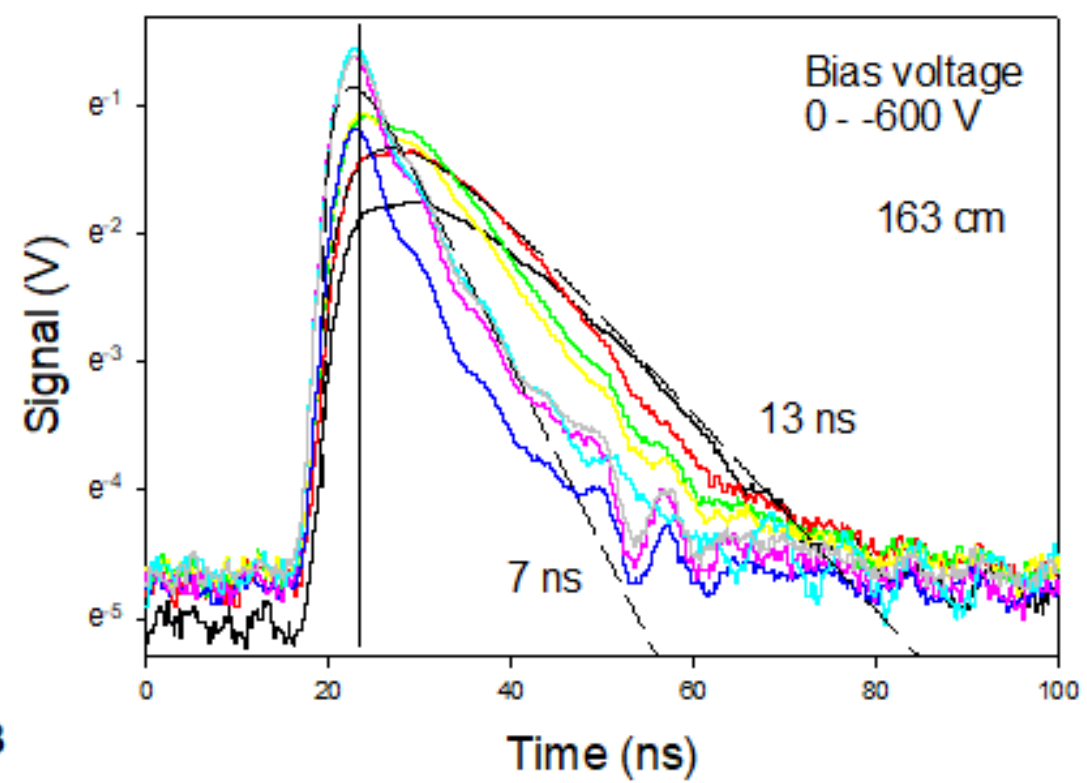

Figure 11

a. Signal from $\mathrm{D}(0)$ to inner collector at $64 \mathrm{~cm}$. Dashed curves are calculated. Variation of collector bias as $0,-50,-100,-200,-300,-400,-500$ and $-600 \mathrm{~V}$. The signal limiting behavior at large negative voltage indicates that secondary electron emission dominates over pair formation. b. Signal from $D(0)$ to outer collector at $163 \mathrm{~cm}$. Dashed curves are calculated. Variation of collector bias as $0,-50,-100,-200,-300$, $-400,-500$ and $-600 \mathrm{~V}$. The signal limiting behavior at large negative voltage indicates that secondary electron emission dominates over pair formation. 


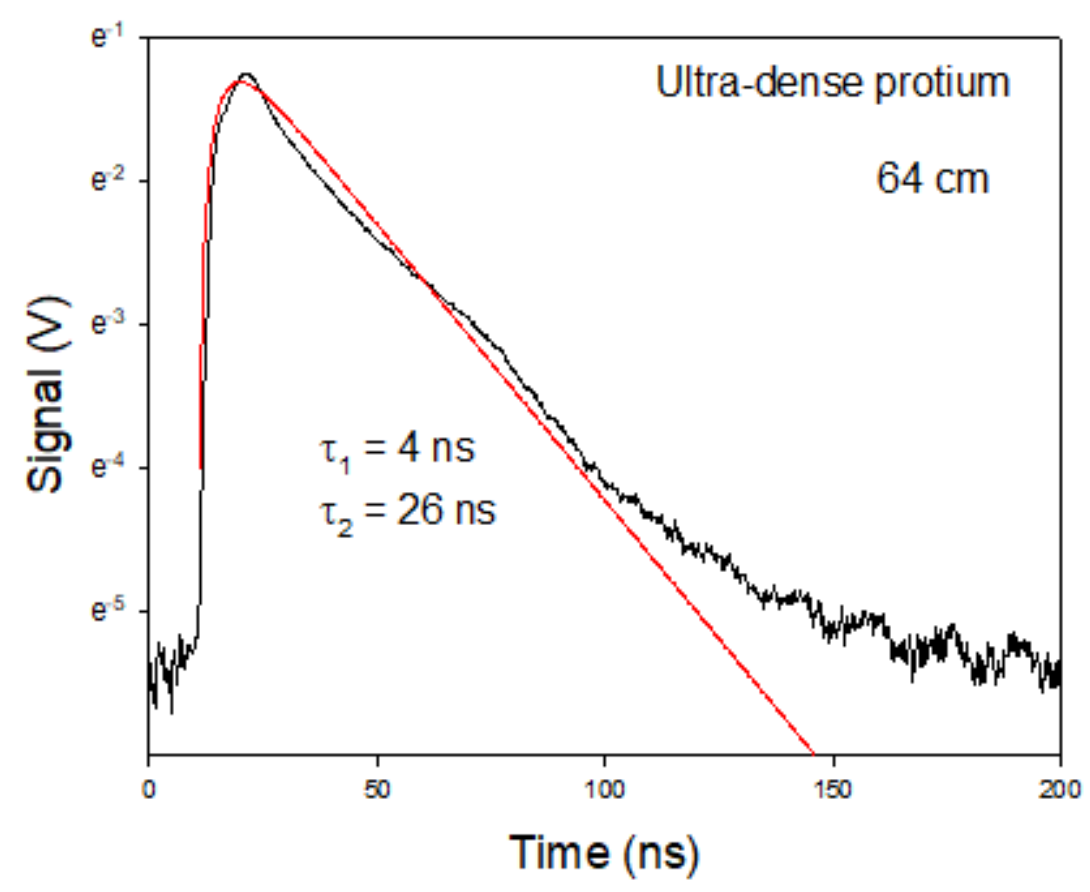

Figure 12

Signal from $\mathrm{p}(0)$ to inner collector at $64 \mathrm{~cm}$ with bias $-24 \mathrm{~V}$. Full curve is calculated with $\tau 1=4 \mathrm{~ns}$ and $\tau 2$ $=26 \mathrm{~ns}$.

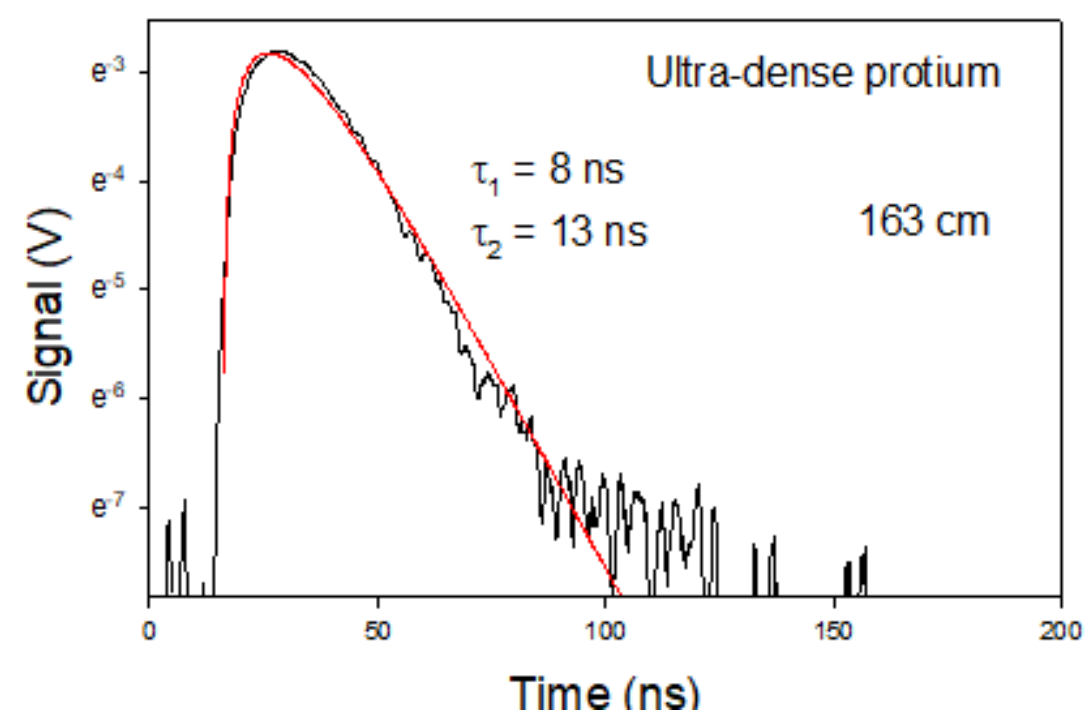

Figure 13

Signal from $\mathrm{p}(0)$ to outer collector at $163 \mathrm{~cm}$ with bias $-24 \mathrm{~V}$. Full curve is calculated with $\tau 1=8 \mathrm{~ns}$ and $\tau 2$ $=13 \mathrm{~ns}$. 


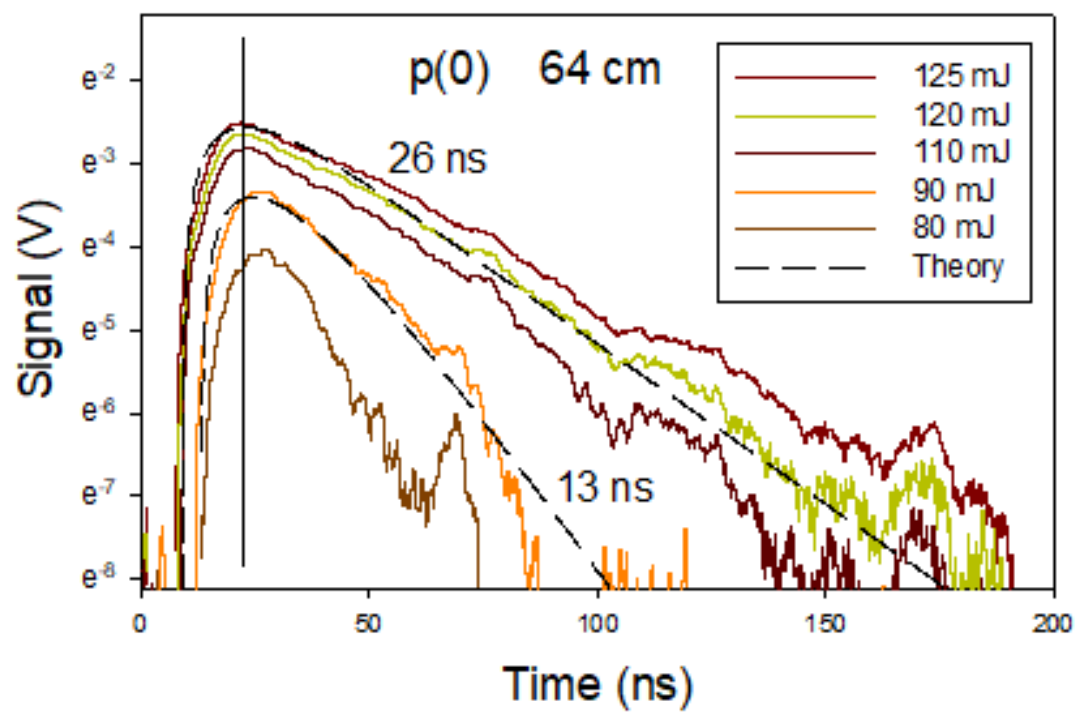

\section{Figure 14}

Signal from $\mathrm{p}(0)$ to inner collector at $64 \mathrm{~cm}$ with bias $-24 \mathrm{~V}$. The laser pulse-energy is varied as shown. The dashed curves are calculated. Upper curve $\tau 1=8 \mathrm{~ns}$ and $\tau 2=26 \mathrm{~ns}$, and lower curve $\tau 1=10 \mathrm{~ns}$ and $\tau 2=13 \mathrm{~ns}$. A narrow slit was used at the exit from the target chamber.

\section{Supplementary Files}

This is a list of supplementary files associated with this preprint. Click to download.

- appendix.docx 